\title{
Palladium-Catalyzed Domino Heck/Sulfination: Synthesis of Sulfonylated Hetero- and Carbocyclic Scaffolds Using DABCO- Bis(sulfur dioxide)
}

Jonathan Bajohr, Abdoul G. Dialllo ${ }^{\dagger}$, Andrew Whyte, Sylvain Gaillard ${ }^{\dagger}, \underline{\text { Jean-Luc Renaud }}^{\dagger^{*}}$, and Mark Lautens*

mark.1autens@,utoronto.ca

jean-luc.renaud@ensicaen.fr

Department of Chemistry, University of Toronto, 80 St. George Street, Toronto, Ontario, M5S 3H6, Canada

${ }^{\dagger}$ Normandie Univ., LCMT, ENSICAEN, UNICAEN, CNRS, 14000 Caen, France

\section{Supporting Information}

\section{Contents}

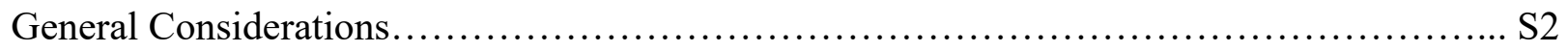

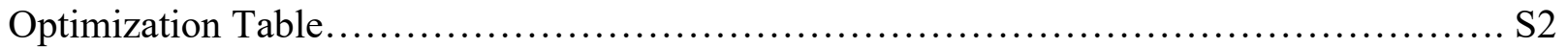

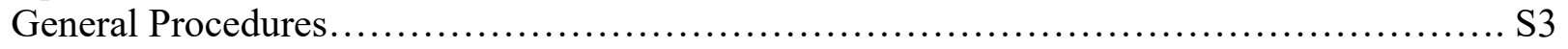

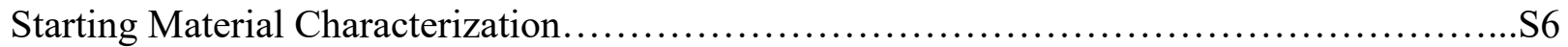

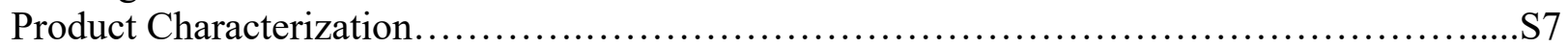

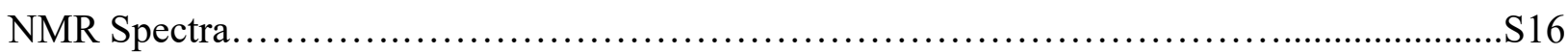

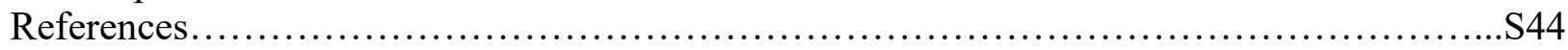




\section{General Considerations}

Reactions were performed under argon in a dry environment unless otherwise stated. Reaction progress was monitored by thin layer chromatography (TLC) and visualized under UV light or CAM stain. Triethylamine was distilled prior to use. 1,3,5- Trimethoxybenzene was purchased from Combi-Blocks and used as received. DABSO was synthesized according to the preparation outlined by Borggraeve and coworkers, and matched all reported spectroscopic data. ${ }^{1}$ All other starting materials and reagents were purchased from Sigma, Alfa Aesar, Fisher, or Combi-Blocks and were used as received. Catalytic reactions were performed in 2 dram vials equipped with a teflon septa (ThermoScientific National B7995-15) and a stir bar (Fisher cat no. 14-513-57, $12 \mathrm{x}$ $4.5 \mathrm{~mm}$ ). An oil bath was used as the heating source for reactions requiring heat. Flash column chromatography was performed with Silicycle 46-60 $\mu \mathrm{m}$ silica gel.

${ }^{1} \mathrm{H}$ and ${ }^{13} \mathrm{C}$ NMR were obtained at $296 \mathrm{~K}$ on Agilent DD2 500 equipped with a $5 \mathrm{~mm}$ Xses Cold Probe. ${ }^{19} \mathrm{~F}$ NMR were obtained on a Bruker Avance III 400. Measurements were referenced to the solvent. NMR data are referenced as chemical shift $(\delta \mathrm{ppm})$, multiplicity $(\mathrm{s}=$ singlet, $\mathrm{d}=$ doublet, $\mathrm{t}=$ triplet, $\mathrm{q}=$ quartet, $\mathrm{m}=$ multiplet, $\mathrm{b}=$ broad), coupling constant $(\mathrm{Hz})$, integration. NMR yields were obtained by ${ }^{1} \mathrm{H}$ NMR analysis using a 10 second relaxation delay and 1,3,5trimethoxybenzene as an internal standard. HRMS were obtained on a JEOL AccuTOF-DART performed at the Advanced Instrumentation for Molecular Structure (AIMS) at the University of Toronto. IR spectra were aquired on a Perkin-Elmer Spectrum 100 instrument with a singlebounce diamond/ZeSe ATR accessory. Data is presented in wavenumbers $\left(\mathrm{cm}^{-1}\right)$.

\section{Optimization Table}

Table S1: Reaction Optimization

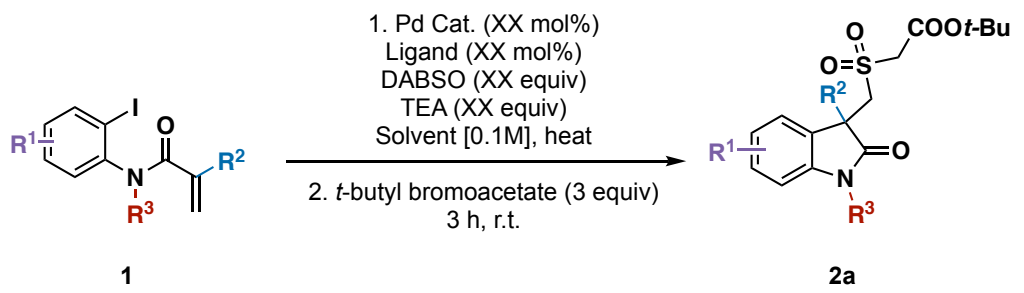

\begin{tabular}{|c|c|c|c|c|c|c|c|}
\hline $\mathrm{Pd}$ & Ligand & $\begin{array}{l}\text { DABSO } \\
\text { (equiv) }\end{array}$ & $\begin{array}{c}\text { TEA } \\
\text { (equiv) }\end{array}$ & Solvent & Temp. $\left({ }^{\circ} \mathrm{C}\right)$ & Additive & $\begin{array}{c}\text { Yield 2a } \\
(\%)^{\mathrm{a}}\end{array}$ \\
\hline $\begin{array}{c}\mathrm{Pd}(\mathrm{OAc})_{2} \\
(5 \mathrm{~mol} \%)\end{array}$ & $\begin{array}{c}\mathrm{PPh}_{3} \\
\left(10 \mathrm{~mol}^{\circ}\right)\end{array}$ & 1.5 & 2 & $\mathrm{MeCN}$ & 80 & none & 58 \\
\hline $\begin{array}{l}\mathrm{Pd}(\mathrm{OAc})_{2} \\
(5 \mathrm{~mol} \%)\end{array}$ & $\begin{array}{c}\mathrm{PPh}_{3} \\
\left(10 \mathrm{~mol}^{\circ}\right)\end{array}$ & 1.5 & 0 & $\mathrm{MeCN}$ & 80 & none & 0 \\
\hline $\begin{array}{l}\operatorname{Pd}(\mathrm{OAc})_{2} \\
(5 \mathrm{~mol} \%)\end{array}$ & $\begin{array}{c}\mathrm{PPh}_{3} \\
\left(10 \mathrm{~mol}^{2}\right)\end{array}$ & 1.5 & 2 & DMF & 80 & none & $77(74)$ \\
\hline $\begin{array}{l}\mathrm{Pd}(\mathrm{OAc})_{2} \\
(5 \mathrm{~mol} \%)\end{array}$ & none & 1.5 & 2 & DMF & 80 & none & 74 \\
\hline $\begin{array}{l}\mathrm{Pd}\left(\mathrm{PPh}_{3}\right)_{4} \\
\left(5 \mathrm{~mol}_{\%}\right)\end{array}$ & none & 1.5 & 2 & DMF & 80 & none & 62 \\
\hline
\end{tabular}




\begin{tabular}{|c|c|c|c|c|c|c|c|}
\hline $\begin{array}{c}\mathrm{Pd}(\mathrm{OAc})_{2} \\
(5 \mathrm{~mol} \%)\end{array}$ & $\begin{array}{c}\mathrm{PPh}_{3} \\
\left(10 \mathrm{~mol}_{\%}\right)\end{array}$ & 1.5 & 2 & DMF & 80 & $20 \mu \mathrm{L} \mathrm{H}_{2} \mathrm{O}$ & 64 \\
\hline $\begin{array}{c}\mathrm{Pd}(\mathrm{OAc})_{2} \\
(5 \mathrm{~mol} \%)\end{array}$ & $\begin{array}{c}\mathrm{PPh}_{3} \\
\left(10 \mathrm{~mol}_{0}\right)\end{array}$ & 1.5 & 2 & $\mathrm{DMF}$ & 90 & none & 77 \\
\hline $\begin{array}{c}\mathrm{Pd}(\mathrm{OAc})_{2} \\
(5 \mathrm{~mol} \%)\end{array}$ & $\begin{array}{c}\mathrm{PPh}_{3} \\
\left(10 \mathrm{~mol}^{2}\right)\end{array}$ & 1.5 & 1 & $\mathrm{DMF}$ & 80 & none & 54 \\
\hline $\begin{array}{c}\mathrm{Pd}(\mathrm{OAc})_{2} \\
(5 \mathrm{~mol} \%)\end{array}$ & $\begin{array}{c}\mathrm{PPh}_{3} \\
\left(10 \mathrm{~mol}_{\%}\right)\end{array}$ & 0.5 & 2 & DMF & 80 & none & 44 \\
\hline
\end{tabular}

Table S1. All reactions performed on a $0.2 \mathrm{mmol}$ scale. NMR yields acquired using 1,3,5-trimethoxybenzene as internal standard. a Isolated yields shown in parentheses.

\section{General Procedures}

\section{General Procedure 1 (GP1): Acylation of anilines and methylation of acrylamides}

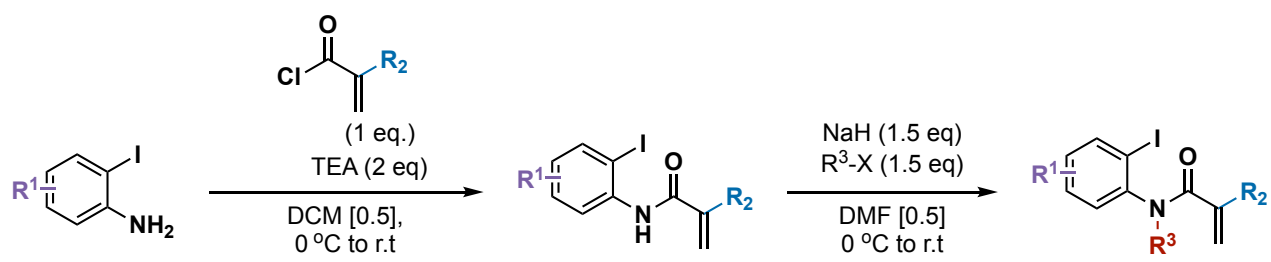

\section{Scheme S1}

General procedure for the synthesis of compounds $\mathbf{1 a - 1 k}$, following a modified procedure from the literature ${ }^{2,3}$ :

Reactions typically performed on a 2-10 mmol scale. A flame dried round bottom flask, under argon, was charged with substituted aniline (1 equiv), dry DCM [0.5 M], and triethylamine (2 equiv) and brought to $0{ }^{\circ} \mathrm{C}$. Then, methacryloyl chloride or 2-phenyl-2-propenoyl chloride (1 equiv) was added dropwise to the solution. The reaction was allowed to warm to room temperature and monitored by TLC. Following the consumption of the starting materials, the crude reaction mixture was washed with water and extracted with DCM $(3 \times 10 \mathrm{~mL})$. The organic layers were combined, dried over $\mathrm{MgSO}_{4}$ and concentrated in vacuo to give crude $\mathrm{NH}-$ acrylamide and used in the subsequent step without further purification.

To a solution of crude acrylamide (1 equiv) in DMF $[0.5 \mathrm{M}]$ at $0{ }^{\circ} \mathrm{C}$ was added $\mathrm{NaH}(60 \%$ dispersion in mineral oil 1.5 equiv) in portions. The resulting slurry was left to stir for 30 minutes at $0{ }^{\circ} \mathrm{C}$. Then, iodomethane or benzylbromide (1.5 equiv) was added dropwise. The solution was then warmed to room temperature and stirred overnight. The reaction mixture was then quenched with the addition of sat. $\mathrm{NH}_{4} \mathrm{Cl}$ solution, and extracted with EtOAc $(3 \times 10 \mathrm{~mL})$. The combined organic layers were dried over $\mathrm{MgSO}_{4}$ and concentrated in vacuo. The crude residues were purified by column chromatography (silica gel, EtOAc/Pentanes) to give acrylamide substrates $\mathbf{1 a - 1 k}$. 


\section{General Procedure 2 (GP2): Alkylation of phenols}

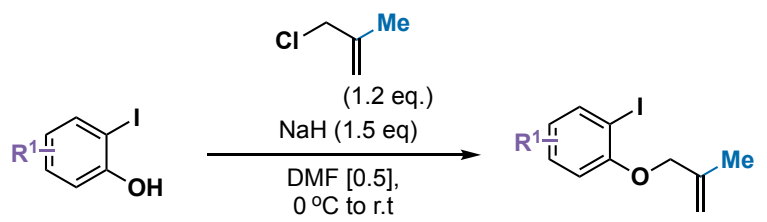

Scheme S2

General procedure for the synthesis of compounds 3a-3c, following a modified preparation from the literature ${ }^{4}$ :

Reactions typically performed on a 2-10 mmol scale. To a flame dried, round bottom flask, under argon, was charged with phenol (1 equiv) and DMF $[0.5 \mathrm{M}]$ and brought to $0{ }^{\circ} \mathrm{C}$. Then, $\mathrm{NaH}$ (60\% dispersion in mineral oil, 1.5 equiv) was added in portions and the resulting slurry left to stir at $0{ }^{\circ} \mathrm{C}$ for 30 minutes. Methallyl chloride (1.2 equiv) was then added to the solution dropwise. The reaction was warmed to room temperature and left to stir overnight. The reaction was quenched with water and extracted with EtOAc $(3 \times 20 \mathrm{~mL})$, then the volatiles were combined, dried over $\mathrm{MgSO}_{4}$, and concentrated in vacuo. The crude materials were purified by column chromatography (silica gel, DCM/Pentanes) to afford substrates 3a-3c.

\section{General Procedure 3 (GP3): Synthesis of sulfone-containing heterocycles}

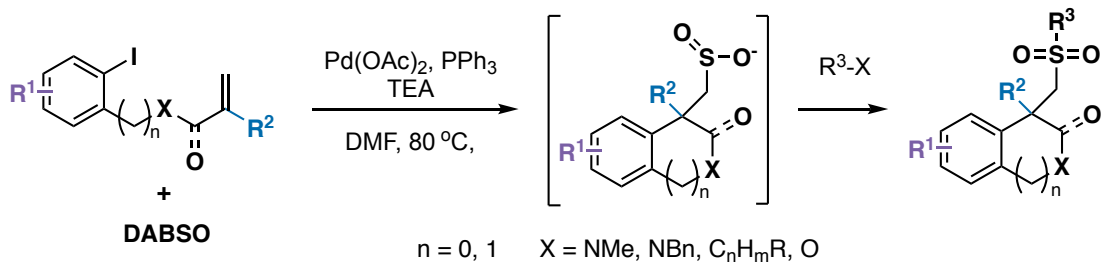

To an oven dried, 2 dram vial containg an oven dried stir bar, under argon, was charged aryl iodide (0.2 mmol, 1 equiv), $\mathrm{Pd}(\mathrm{OAc})_{2}(5 \mathrm{~mol} \%), \mathrm{PPh}_{3}(10 \mathrm{~mol} \%)$ and DABSO (0.3 mmol, 1.5 equiv). Meanwhile, TEA ( $0.4 \mathrm{mmol}, 2$ equiv) was added to a syringe of anhydrous DMF ( $2 \mathrm{~mL})$. Following addition of base and solvent, the reactions were left to stir for 16 hours at $80{ }^{\circ} \mathrm{C}$. Then, the resulting mixture was cooled to room temperature before adding $t$-butylbromoacetate $(0.6$ mmol, 3 equiv) or $m$-nitrobenzyl bromide ( $0.6 \mathrm{mmol}, 3$ equiv). The reactions were left to stir at room temperature for an additional 3 hours. The reactions were diluted with EtOAc $(5 \mathrm{~mL})$ and washed with a $10 \%$ by weight solution of $\mathrm{LiCl}$ in $\mathrm{H}_{2} \mathrm{O}(2 \times 3 \mathrm{~mL})$. The organic layer was concentrated in vacuo and purified by column chromatography on silica gel to give the final sulfone products. 


\section{General Procedure 4 (GP4): Synthesis of sulfonamides}

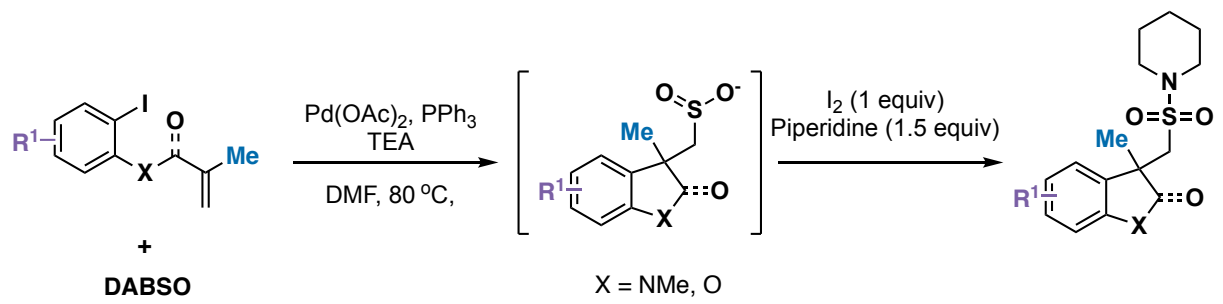

To an oven dried, 2 dram vial containg an oven dried stir bar, under argon, was charged aryl iodide (0.2 mmol, 1 equiv), $\mathrm{Pd}(\mathrm{OAc})_{2}(5 \mathrm{~mol} \%), \mathrm{PPh}_{3}(10 \mathrm{~mol} \%)$ and DABSO (0.3 mmol, 1.5 equiv). Meanwhile, TEA ( $0.4 \mathrm{mmol}, 2$ equiv) was added to a syringe of anhydrous DMF ( $2 \mathrm{~mL})$. Following addition of base and solvent, the reactions were left to stir for 16 hours at $80{ }^{\circ} \mathrm{C}$. Then, the resulting mixture was cooled to room temperature before adding $\mathrm{I}_{2}$ ( 1 equiv) and piperidine ( $0.3 \mathrm{mmol}, 1.5$ equiv) and letting stir for 18 hours at room temperature. The reactions were diluted with EtOAc $(5 \mathrm{~mL})$ and washed with a $10 \%$ by weight solution of $\mathrm{LiCl}$ in $\mathrm{H}_{2} \mathrm{O}(2 \mathrm{x} 3 \mathrm{~mL})$. The organic layer was concentrated in vacuo and purified by column chromatography on silica gel to give the final sulfonamide products.

\section{General Procedure 5 (GP5): Synthesis of sulfonyl fluorides}

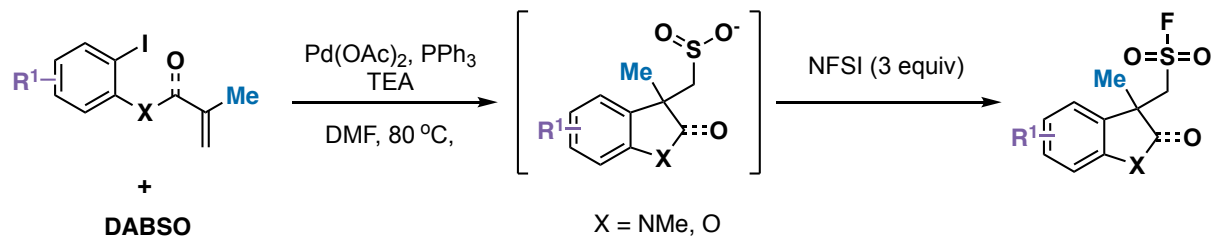

To an oven dried, 2 dram vial containg an oven dried stir bar, under argon, was charged aryl iodide (0.2 mmol, 1 equiv), $\mathrm{Pd}(\mathrm{OAc})_{2}(5 \mathrm{~mol} \%), \mathrm{PPh}_{3}(10 \mathrm{~mol} \%)$ and DABSO (0.3 mmol, 1.5 equiv). Meanwhile, TEA ( $0.4 \mathrm{mmol}, 2$ equiv) was added to a syringe of anhydrous DMF ( $2 \mathrm{~mL})$. Following addition of base and solvent, the reactions were left to stir for 16 hours at $80{ }^{\circ} \mathrm{C}$. Then, the resulting mixture was cooled to room temperature before adding NFSI ( 3 equiv) dissolved in DMF $(0.4 \mathrm{~mL})$ dropwise at $0{ }^{\circ} \mathrm{C}$. The reaction was left to stir at $0{ }^{\circ} \mathrm{C}$ and allowed to slowly warm to room temperature after 3 hours. The reactions were diluted with EtOAc $(5 \mathrm{~mL})$ and washed with a $10 \%$ by weight solution of $\mathrm{LiCl}$ in $\mathrm{H}_{2} \mathrm{O}(2 \times 3 \mathrm{~mL})$. The organic layer was concentrated in vacuo and purified by column chromatography on silica gel to give the final sulfonyl fluoride products. 


\section{Known Starting Material}

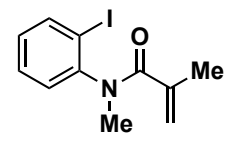

$1 \mathrm{a}$

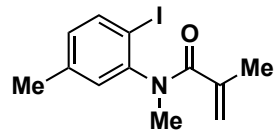

$1 b$

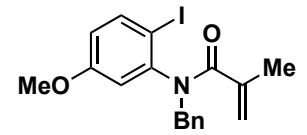

1c

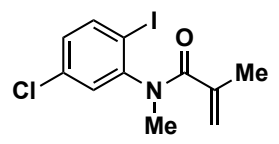

$1 d$

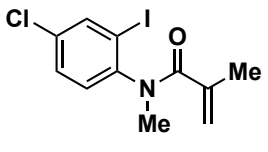

$1 \mathrm{e}$

Refer to Y. Feng, S. Yang, S. Zhao, D. P. Zhang, X. Li, H. Liu, Y.Dong, F. G. Sun, Org. Lett. 2020, 22, 6734
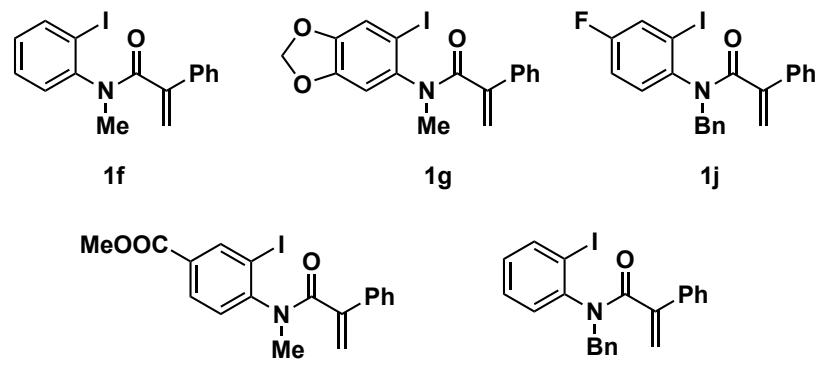

$1 \mathrm{k}$

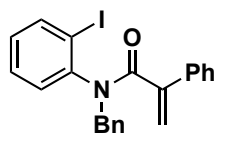

$1 \mathrm{~h}$

Refer to H. Yoon, A. Lossouarn, F. Landau, M. Lautens, Org. Lett. 2016, 18, 6324

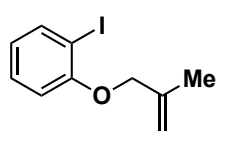

3a

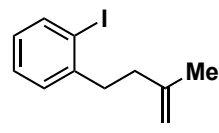

$3 e$<smiles>CC(C)=CCOc1ccc(Cl)cc1I</smiles>

3b

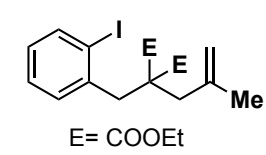

$3 f$

3a and 3e: Refer to J. He, Y. Xue, B. Han, C. Zhang, Y. Wang, S. Zhu, Angew. Chem. Int. Ed. 2020, 59, 2328 [ref 4]

3b Refer to Y. Gao, W. Xiong, H. Chen, W. Wu, J. Peng, Y. Gao, H. Jiang, J. Org. Chem. 2015, 80, 7456 [ref 5]

3d Refer to M. Wollenburg, J. Bajohr, A. D. Marchese, A. Whyte, F. Glorius, M. Lautens, Org. Lett. 2020, 22, 3679 [ref 6] 
3f Refer to Z. Lu, C. Hu, J. Guo, J. Li, Y. Cui, Y. Jia, Org. Lett 2010, 12, 480 [ref 7]

New Starting Material

1-iodo-3-methoxy-2-((2-methylallyl)oxy)benzene (3c)<smiles>C=C(C)COc1c(I)cccc1OC</smiles>

Synthesized according to GP2. To a solution of 2-iodo-6-methoxyphenol (5 mmol, 1 equiv) in DMF $(10 \mathrm{~mL})$ at $0{ }^{\circ} \mathrm{C}$ was added $\mathrm{NaH}(60 \%$ dispersion in mineral oil, 1.5 equiv) and allowed to stir for 30 minutes at $0{ }^{\circ} \mathrm{C}$. Then, methallyl chloride ( 1.2 equiv) was added dropwise and the solution allowed to stir at room temperature overnight. The reaction was quenched with water and extracted with EtOAc $(3 \times 20 \mathrm{~mL})$, then the volatiles were combined, dried over $\mathrm{MgSO}_{4}$, and concentrated in vacuo. The crude material was purified by column chromatography (silica gel, DCM/Pentanes $0 \%$-> 30\%) to afford substrate $3 \mathrm{c}$ as a colorless oil (1.26 g, 83\% yield).

${ }^{1} \mathbf{H}$ NMR $\left(500 \mathrm{MHz}, \mathrm{CDCl}_{3}\right) \delta 7.35(\mathrm{dd}, J=7.9,1.4 \mathrm{~Hz}, 1 \mathrm{H}), 6.88(\mathrm{dd}, J=8.2,1.4 \mathrm{~Hz}, 1 \mathrm{H}), 6.79$ (t, $J=8.0 \mathrm{~Hz}, 1 \mathrm{H}), 5.20(\mathrm{dh}, J=2.1,1.0 \mathrm{~Hz}, 1 \mathrm{H}), 5.00(\mathrm{dtq}, J=2.2,1.5,0.7 \mathrm{~Hz}, 1 \mathrm{H}), 4.40(\mathrm{p}, J$ $=0.6 \mathrm{~Hz}, 2 \mathrm{H}), 3.83(\mathrm{~s}, 3 \mathrm{H}), 1.96(\mathrm{t}, J=1.2 \mathrm{~Hz}, 3 \mathrm{H})$.

${ }^{13} \mathrm{C} \mathrm{NMR}\left(126 \mathrm{MHz}, \mathrm{CDCl}_{3}\right) \delta 152.9,147.9,141.5,130.7,125.8,113.3,112.9,92.8,76.3,56.0$, 20.1 .

IR (ATR) 1699, 1666, 1586, 1473, 1437, 1297, 1280, 1228, 1085, 1035

HRMS (DART) m/z: [M+NH 4$]+$ Calculated for $\mathrm{C}_{11} \mathrm{H}_{17} \mathrm{NO}_{2} \mathrm{I} 322.0299$; Found 322.0300

\section{Product Characterization}

tert-butyl 2-(((1,3-dimethyl-2-oxoindolin-3-yl)methyl)sulfonyl)acetate (2a)

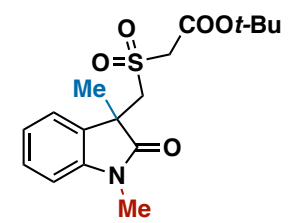

Synthesized according to GP3 from 1a. Purified by column chromatography (silica gel, 0\% -> 40\% EtOAc/Pentane) to afford product $\mathbf{2 a}$ as a clear, sticky sap (52 mg, 74\% yield).

${ }^{1} \mathbf{H}$ NMR $\left(500 \mathrm{MHz}, \mathrm{CDCl}_{3}\right) \delta 7.40-7.32(\mathrm{~m}, 2 \mathrm{H}), 7.11-7.06(\mathrm{~m}, 1 \mathrm{H}), 6.90$ $(\mathrm{d}, \mathrm{J}=7.8,0.8 \mathrm{~Hz}, 1 \mathrm{H}), 4.11(\mathrm{~d}, \mathrm{~J}=15.0 \mathrm{~Hz}, 1 \mathrm{H}), 3.83(\mathrm{dd}, \mathrm{J}=15.0,1.5 \mathrm{~Hz}, 1 \mathrm{H}), 3.58(\mathrm{dd}, \mathrm{J}=$ $14.9,1.5 \mathrm{~Hz}, 1 \mathrm{H}), 3.27(\mathrm{~d}, \mathrm{~J}=14.9 \mathrm{~Hz}, 1 \mathrm{H}), 3.24(\mathrm{~s}, 3 \mathrm{H}), 1.49(\mathrm{~s}, 9 \mathrm{H}), 1.46(\mathrm{~s}, 3 \mathrm{H})$.

${ }^{13}$ C NMR (126 MHz, $\left.\mathrm{CDCl}_{3}\right) \delta 177.8,162.3,143.6,129.9,129.2,123.5,122.5,108.9,84.1$, 59.9, 58.7, 45.5, 27.8, 26.6, 24.9 .

IR (ATR) 2984, 1711, 1622, 1502, 1479, 1380, 1321, 1258, 1130, 957

HRMS (DART) m/z: [M+H]+ Calculated for $\mathrm{C}_{17} \mathrm{H}_{24} \mathrm{NO}_{5} \mathrm{~S}$ 354.1370; Found 354.1374 
tert-butyl 2-(((1,3,6-trimethyl-2-oxoindolin-3-yl)methyl)sulfonyl)acetate (2b)

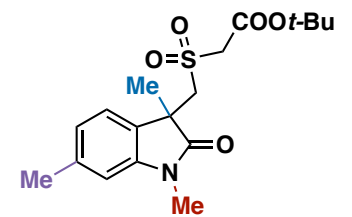

Synthesized according to GP3 from 1b. Purified by column chromatography (silica gel, $0 \%->40 \%$ EtOAc/Pentane) to afford product $\mathbf{2 b}$ as a white solid (54 $\mathrm{mg}, 73 \%$ yield).

${ }^{1} \mathbf{H}$ NMR $\left(500 \mathrm{MHz}, \mathrm{CDCl}_{3}\right) \delta 7.25(\mathrm{~d}, \mathrm{~J}=7.5 \mathrm{~Hz}, 1 \mathrm{H}), 6.91-6.85(\mathrm{~m}$, $1 \mathrm{H}), 6.72(\mathrm{~s}, 1 \mathrm{H}), 4.09(\mathrm{~d}, \mathrm{~J}=15.0 \mathrm{~Hz}, 1 \mathrm{H}), 3.80(\mathrm{dd}, \mathrm{J}=14.9,1.5 \mathrm{~Hz}, 1 \mathrm{H}), 3.57$ (dd, J = 14.9, $1.5 \mathrm{~Hz}, 1 \mathrm{H}), 3.31(\mathrm{~d}, \mathrm{~J}=14.8 \mathrm{~Hz}, 1 \mathrm{H}), 3.22(\mathrm{~s}, 3 \mathrm{H}), 2.39(\mathrm{~s}, 3 \mathrm{H}), 1.49$ (s, 9H), 1.43 (s, 3H). ${ }^{13} \mathrm{C}$ NMR $\left(126 \mathrm{MHz}, \mathrm{CDCl}_{3}\right) \delta 178.1,162.3,143.7,139.5,126.8,123.2,123.0,109.9,84.1$, $59.9,58.7,45.3,27.8,26.6,25.0,21.9$.

IR (ATR) 2987, 2936, 1738, 1717, 1622, 1479, 1380, 1333, 1139, 820

HRMS (DART) m/z: [M+H]+ Calculated for $\mathrm{C}_{18} \mathrm{H}_{26} \mathrm{NO}_{5} \mathrm{~S} 368.1526$; Found 368.1523

MP $114-115^{\circ} \mathrm{C}$

tert-butyl 2-(((6-methoxy-1,3-dimethyl-2-oxoindolin-3-yl)methyl)sulfonyl)acetate (2c)

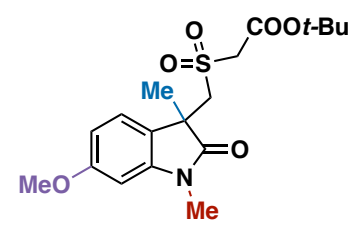

Synthesized according to GP3from 1c. Purified by column chromatography (silica gel, $0 \%->40 \%$ EtOAc/Pentane) to afford product 2c as a white solid (50 $\mathrm{mg}, 65 \%$ yield).

${ }^{1} \mathbf{H}$ NMR $\left(500 \mathrm{MHz}, \mathrm{CDCl}_{3}\right) \delta 7.28-7.26(\mathrm{~m}, 1 \mathrm{H}$, one peak is hidden under the solvent signal), $6.58(\mathrm{dd}, J=8.2,2.3 \mathrm{~Hz}, 1 \mathrm{H}), 6.47(\mathrm{~d}, J=2.3 \mathrm{~Hz}, 1 \mathrm{H}), 4.08(\mathrm{~d}, J=$ $15.0 \mathrm{~Hz}, 1 \mathrm{H}), 3.84$ (s, 3H), 3.77 (dd, $J=15.0,1.2 \mathrm{~Hz}, 1 \mathrm{H}), 3.57$ (dd, $J=14.8,1.5 \mathrm{~Hz}, 1 \mathrm{H}), 3.31$ $(\mathrm{d}, J=14.8 \mathrm{~Hz}, 1 \mathrm{H}), 3.22(\mathrm{~s}, 3 \mathrm{H}), 1.50(\mathrm{~s}, 9 \mathrm{H}), 1.43(\mathrm{~s}, 3 \mathrm{H})$.

${ }^{13} \mathbf{C ~ N M R}\left(126 \mathrm{MHz}, \mathrm{CDCl}_{3}\right) \delta 178.4,162.3,160.9,145.0,124.2,121.5,106.5,96.7,84.1,59.9$, 58.7, 55.5, 45.0, 27.9, 26.6, 25.0.

IR (ATR) 2978, 2915, 1723, 1616, 1371, 1333, 1240, 1136, 1106, 823

HRMS (DART) m/z: [M+H]+ Calculated for $\mathrm{C}_{18} \mathrm{H}_{26} \mathrm{NO}_{6} \mathrm{~S} 384.1475$; Found 384.1450 MP $100-102{ }^{\circ} \mathrm{C}$ tert-butyl 2-(((6-chloro-1,3-dimethyl-2-oxoindolin-3-yl)methyl)sulfonyl)acetate (2d)

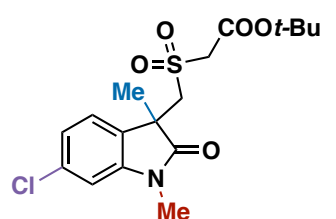

Synthesized according to GP3 from 1d. Purified by column chromatography (silica gel, $0 \%->40 \%$ EtOAc/Pentane) to afford product 2d as a white solid (62 $\mathrm{mg}, 80 \%$ yield).

${ }^{1} \mathbf{H}$ NMR $\left(500 \mathrm{MHz}, \mathrm{CDCl}_{3}\right) \delta 7.31(\mathrm{~d}, J=7.9 \mathrm{~Hz}, 1 \mathrm{H}), 7.07(\mathrm{dd}, J=7.9$, $1.8 \mathrm{~Hz}, 1 \mathrm{H}), 6.90(\mathrm{~d}, J=1.9 \mathrm{~Hz}, 1 \mathrm{H}), 4.03(\mathrm{~d}, J=14.9 \mathrm{~Hz}, 1 \mathrm{H}), 3.87(\mathrm{dd}, J=14.8,1.3 \mathrm{~Hz}, 1 \mathrm{H})$, $3.68(\mathrm{dd}, J=14.8,1.3 \mathrm{~Hz}, 1 \mathrm{H}), 3.41(\mathrm{~d}, J=14.7 \mathrm{~Hz}, 1 \mathrm{H}), 3.23(\mathrm{~s}, 3 \mathrm{H}), 1.50(\mathrm{~s}, 9 \mathrm{H}), 1.45(\mathrm{~s}, 3 \mathrm{H})$. ${ }^{13} \mathrm{C}$ NMR $\left(126 \mathrm{MHz}, \mathrm{CDCl}_{3}\right) \delta 177.8,162.2,144.8,135.0,128.4,124.5,122.4,109.6,84.4$, $60.3,58.5,45.2,27.8,26.7,25.0$.

IR (ATR) 2978, 2939, 1720, 1609, 1502, 1368, 1326, 1240, 1156, 1106

HRMS (DART) m/z: [M+H]+ Calculated for $\mathrm{C}_{17} \mathrm{H}_{23} \mathrm{NO}_{5} \mathrm{SCl} 388.0980$; Found 388.0981 MP $44-45^{\circ} \mathrm{C}$ 
tert-butyl 2-(((5-chloro-1,3-dimethyl-2-oxoindolin-3-yl)methyl)sulfonyl)acetate (2e)

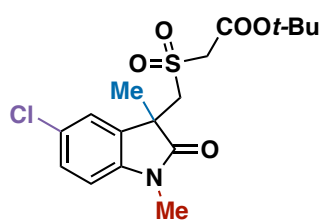

Synthesized according to GP3 from 1e. Purified by column chromatography (silica gel, $0 \%->40 \%$ EtOAc/Pentane) to afford product 2e as a clear, sticky solid (55 mg, 71\% yield).

${ }^{1} \mathbf{H}$ NMR $\left(500 \mathrm{MHz}, \mathrm{CDCl}_{3}\right) \delta 7.37(\mathrm{~d}, J=2.1 \mathrm{~Hz}, 1 \mathrm{H}), 7.31(\mathrm{dd}, J=8.3$, $2.1 \mathrm{~Hz}, 1 \mathrm{H}), 6.82(\mathrm{~d}, J=8.3 \mathrm{~Hz}, 1 \mathrm{H}), 4.04(\mathrm{~d}, J=15.0 \mathrm{~Hz}, 1 \mathrm{H}), 3.89(\mathrm{dd}, J=14.9,1.2 \mathrm{~Hz}, 1 \mathrm{H})$, $3.70(\mathrm{dd}, J=14.9,1.2 \mathrm{~Hz}, 1 \mathrm{H}), 3.37(\mathrm{~d}, J=14.9 \mathrm{~Hz}, 1 \mathrm{H}), 3.23(\mathrm{~s}, 3 \mathrm{H}), 1.51(\mathrm{~s}, 9 \mathrm{H}), 1.46(\mathrm{~s}, 3 \mathrm{H})$. ${ }^{13}$ C NMR $\left(126 \mathrm{MHz}, \mathrm{CDCl}_{3}\right) \delta 177.3,162.1,142.1,131.8,129.1,128.0,124.1,109.8,84.4$, 60.1, 58.5, 45.7, 27.8, 26.7, 24.9.

IR (ATR) 2986, 2933, 1717, 1618, 1496, 1463, 1368, 1329, 1138, 1106, 909, 831

HRMS (DART) m/z: [M+H]+ Calculated for $\mathrm{C}_{17} \mathrm{H}_{23} \mathrm{NO}_{5} \mathrm{SCl} 388.0980$; Found 388.0977 MP $31-32^{\circ} \mathrm{C}$

tert-butyl 2-(((1-methyl-2-oxo-3-phenylindolin-3-yl)methyl)sulfonyl)acetate (2f)

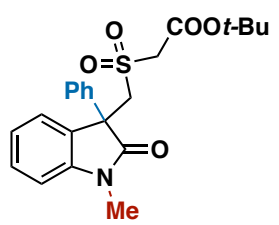

Synthesized according to GP3 from 1f. Purified by column chromatography (silica gel, $0 \%->40 \%$ EtOAc/Pentane) to afford product $\mathbf{2 f}$ as a white solid (63mg, 76\% yield).

${ }^{1} \mathbf{H}$ NMR $\left(500 \mathrm{MHz}, \mathrm{CDCl}_{3}\right) \delta 7.50-7.47(\mathrm{~m}, 1 \mathrm{H}), 7.45-7.37(\mathrm{~m}, 3 \mathrm{H}), 7.35$ $-7.27(\mathrm{~m}, 3 \mathrm{H}), 7.19-7.15(\mathrm{~m}, 1 \mathrm{H}), 6.97-6.94(\mathrm{~m}, 1 \mathrm{H}), 4.46-4.43(\mathrm{~m}, 2 \mathrm{H}), 3.76-3.70(\mathrm{~m}$, $1 \mathrm{H}), 3.30(\mathrm{~d}, J=14.9 \mathrm{~Hz}, 1 \mathrm{H}), 3.24(\mathrm{~s}, 3 \mathrm{H}), 1.50(\mathrm{~s}, 9 \mathrm{H})$.

${ }^{13} \mathrm{C}$ NMR $\left(126 \mathrm{MHz}, \mathrm{CDCl}_{3}\right) \delta 176.2,162.3,144.6,137.7,129.7,129.0,128.2,128.0,126.6$, $125.9,122.4,109.2,84.2,60.3,59.3,52.9,27.9,26.9$.

IR (ATR) 2998, 2983, 2933, 1726, 1705, 1615, 1338, 1147, 1112, 825

HRMS (DART) m/z: [M+H] + Calculated for $\mathrm{C}_{22} \mathrm{H}_{26} \mathrm{NO}_{5} \mathrm{~S} 416.1526$; Found 416.1530 MP $134-136^{\circ} \mathrm{C}$

tert-butyl 2-(((5-methyl-6-oxo-7-phenyl-6,7-dihydro-5H-[1,3]dioxolo[4,5-f]indol-7yl)methyl)sulfonyl)acetate (2g)

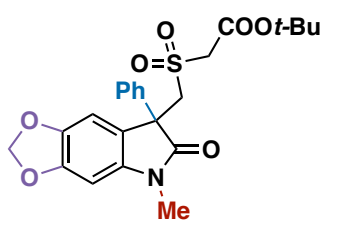

Synthesized according to GP3 from 1g. Purified by column chromatography (silica gel, $0 \%$-> 40\% EtOAc/Pentane) to afford product $2 \mathrm{~g}$ as a white solid (62 $\mathrm{mg}, 67 \%$ yield).

${ }^{1} \mathbf{H}$ NMR $\left(500 \mathrm{MHz}, \mathrm{CDCl}_{3}\right) \delta 7.39-7.26(\mathrm{~m}, 5 \mathrm{H}), 6.97(\mathrm{~s}, 1 \mathrm{H}), 6.55(\mathrm{~s}$, $1 \mathrm{H}), 6.03-5.97(\mathrm{~m}, 2 \mathrm{H}), 4.44(\mathrm{dd}, J=15.0,1.0 \mathrm{~Hz}, 1 \mathrm{H}), 4.34(\mathrm{~d}, J=14.9 \mathrm{~Hz}, 1 \mathrm{H}), 3.82(\mathrm{dd}, J=$ $14.9,1.2 \mathrm{~Hz}, 1 \mathrm{H}), 3.44(\mathrm{~d}, J=14.9 \mathrm{~Hz}, 1 \mathrm{H}), 3.19(\mathrm{~s}, 3 \mathrm{H}), 1.51(\mathrm{~s}, 9 \mathrm{H})$.

${ }^{13} \mathrm{C}$ NMR $\left(126 \mathrm{MHz}, \mathrm{CDCl}_{3}\right) \delta 176.4,162.3,148.6,143.3,139.2,138.0,129.0,128.2,126.5$, $119.5,107.1,101.5,92.9,84.3,60.4,59.2,53.1,27.9,27.1$.

IR (ATR) 2983, 2933, 1717, 1624, 1478, 1389, 1338, 1252, 1141, 1034

HRMS (DART) m/z: [M+H]+ Calculated for $\mathrm{C}_{23} \mathrm{H}_{26} \mathrm{NO}_{7} \mathrm{~S} 460.1426$; Found 460.1422 MP $92-93{ }^{\circ} \mathrm{C}$ 
tert-butyl 2-(((1-benzyl-2-oxo-3-phenylindolin-3-yl)methyl)sulfonyl)acetate (2h)

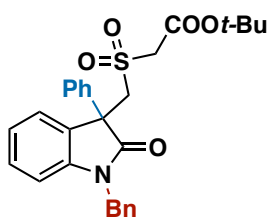

Synthesized according to GP3 from $\mathbf{1 h}$. Purified by column chromatography (silica gel, $0 \%$-> 40\% EtOAc/Pentane) to afford product $\mathbf{2 h}$ as a white solid (69 mg, 70\% yield).

${ }^{1} \mathbf{H}$ NMR $\left(500 \mathrm{MHz}, \mathrm{CDCl}_{3}\right) \delta 7.51-7.47(\mathrm{~m}, 1 \mathrm{H}), 7.42-7.38(\mathrm{~m}, 2 \mathrm{H}), 7.36$ $-7.29(\mathrm{~m}, 4 \mathrm{H}), 7.29-7.26(\mathrm{~m}, 4 \mathrm{H}), 7.26-7.25(\mathrm{~m}, 1 \mathrm{H}), 7.13(\mathrm{td}, J=7.6,1.0 \mathrm{~Hz}, 1 \mathrm{H}), 6.79(\mathrm{dt}$, $J=7.9,0.8 \mathrm{~Hz}, 1 \mathrm{H}), 5.10(\mathrm{~d}, J=15.9 \mathrm{~Hz}, 1 \mathrm{H}), 4.80(\mathrm{~d}, J=15.9 \mathrm{~Hz}, 1 \mathrm{H}), 4.58-4.43(\mathrm{~m}, 2 \mathrm{H})$, $3.82-3.75(\mathrm{~m}, 1 \mathrm{H}), 3.39(\mathrm{~d}, J=14.8 \mathrm{~Hz}, 1 \mathrm{H}), 1.51(\mathrm{~s}, 9 \mathrm{H})$.

${ }^{13}$ C NMR $\left(126 \mathrm{MHz}, \mathrm{CDCl}_{3}\right) \delta 176.3,162.3,143.8,138.0,135.6,129.5,129.1,128.7,128.3$, $128.2,127.6,127.2,126.5,125.8,122.5,110.3,84.3,60.4,59.0,53.0,44.6,27.9$.

IR (ATR) 2989, 1734, 1699, 1615, 1499, 1466, 1371, 1299, 1156, 954

HRMS (DART) m/z: [M+H]+ Calcualted for $\mathrm{C}_{28} \mathrm{H}_{30} \mathrm{NO}_{5} \mathrm{~S} 492.1839$; Found 492.1837

MP $167-170{ }^{\circ} \mathrm{C}$

tert-butyl 2-(((1-methyl-2-oxo-3-(4-(trifluoromethyl)phenyl)indolin-3yl)methyl)sulfonyl)acetate (2i)

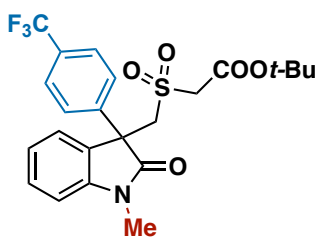

Synthesized according to GP3 from 1i. Purified by column chromatography (silica gel, $0 \%->40 \%$ EtOAc/Pentane) to afford product $\mathbf{2 i}$ as a white solid (67 mg, 71\% yield).

${ }^{1} \mathbf{H}$ NMR $\left(500 \mathrm{MHz}, \mathrm{CDCl}_{3}\right) \delta 7.60-7.56(\mathrm{~m}, 2 \mathrm{H}), 7.55-7.51(\mathrm{~m}, 2 \mathrm{H})$, $7.49(\mathrm{ddd}, J=7.5,1.3,0.6 \mathrm{~Hz}, 1 \mathrm{H}), 7.45(\mathrm{td}, J=7.8,1.2 \mathrm{~Hz}, 1 \mathrm{H}), 7.20(\mathrm{td}, J=7.6,1.0 \mathrm{~Hz}, 1 \mathrm{H})$, $6.98(\mathrm{dt}, J=7.9,0.8 \mathrm{~Hz}, 1 \mathrm{H}), 4.48-4.39(\mathrm{~m}, 2 \mathrm{H}), 3.74(\mathrm{dt}, J=14.9,0.8 \mathrm{~Hz}, 1 \mathrm{H}), 3.32(\mathrm{~d}, J=$ $14.9 \mathrm{~Hz}, 1 \mathrm{H}), 3.25(\mathrm{~s}, 3 \mathrm{H}), 1.50(\mathrm{~s}, 9 \mathrm{H})$.

${ }^{13}$ C NMR $\left(126 \mathrm{MHz}, \mathrm{CDCl}_{3}\right) \delta 175.5,162.3,144.6,141.6,130.6,130.4,130.1,127.2,127.2$, $125.9,123.8(\mathrm{q}, J=277.1 \mathrm{~Hz}), 122.7,109.5,84.4,60.3,59.0,52.8,27.8,27.0$.

${ }^{19}$ F NMR $\left(400 \mathrm{MHz}, \mathrm{CDCl}_{3}\right) \delta 62.8$

IR (ATR) 2989, 1723, 1615, 1472, 1377, 1326, 1165, 1121, 1076, 849

HRMS (DART) m/z: [M+H]+ Calculated for $\mathrm{C}_{23} \mathrm{H}_{25} \mathrm{NO}_{5} \mathrm{~F}_{3} \mathrm{~S} 484.1400$; Found 484.1397 MP $56-60{ }^{\circ} \mathrm{C}$

tert-butyl 2-(((1-benzyl-5-fluoro-2-oxo-3-phenylindolin-3-yl)methyl)sulfonyl)acetate (2j)

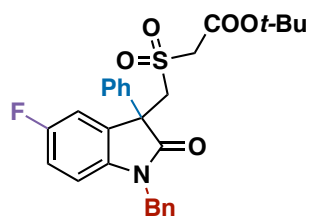

Synthesized according to GP3 from 1j. Purified by column chromatography (silica gel, $0 \%->40 \%$ EtOAc/Pentane) to afford product $\mathbf{2} \mathbf{j}$ as a white solid (70 mg, 69\% yield).

${ }^{1} \mathbf{H}$ NMR $\left(500 \mathrm{MHz}, \mathrm{CDCl}_{3}\right) \delta 7.40-7.26(\mathrm{~m}, 8 \mathrm{H}), 7.26-7.22(\mathrm{~m}, 3 \mathrm{H})$, $6.97(\mathrm{td}, J=8.8,2.6 \mathrm{~Hz}, 1 \mathrm{H}), 6.68(\mathrm{dd}, J=8.7,4.2 \mathrm{~Hz}, 1 \mathrm{H}), 5.06(\mathrm{~d}, J=15.9 \mathrm{~Hz}, 1 \mathrm{H}), 4.81(\mathrm{~d}, J$ $=15.9 \mathrm{~Hz}, 1 \mathrm{H}), 4.59(\mathrm{dd}, J=14.8,1.1 \mathrm{~Hz}, 1 \mathrm{H}), 4.39$ (d, $J=14.9 \mathrm{~Hz}, 1 \mathrm{H}), 3.89(\mathrm{dd}, J=14.8,1.1$ $\mathrm{Hz}, 1 \mathrm{H}), 3.49$ (d, $J=14.9 \mathrm{~Hz}, 1 \mathrm{H}), 1.52(\mathrm{~s}, 9 \mathrm{H})$.

${ }^{13}$ C NMR $\left(126 \mathrm{MHz} \mathrm{CDCl}_{3}\right) \delta 176.0,162.2,158.9(\mathrm{~d}, J=242.0 \mathrm{~Hz}) 139.5(\mathrm{~d}, J=2.1 \mathrm{~Hz})$, $137.5,135.2,130.0(\mathrm{~d}, J=8.1 \mathrm{~Hz}), 129.2,128.8,128.5,127.7,127.2,126.4,115.9$ (d, $J=23.3$ 
$\mathrm{Hz}), 113.9(\mathrm{~d}, J=25.1 \mathrm{~Hz}), 110.8(\mathrm{~d}, J=8.0 \mathrm{~Hz}), 84.5,60.6,58.9,53.4(\mathrm{~d}, J=1.7 \mathrm{~Hz}), 44.7$, 27.9.

${ }^{19} \mathbf{F}$ NMR $\left(400 \mathrm{MHz}, \mathrm{CDCl}_{3}\right) \delta 119.44$

IR (ATR) 2927, 1734, 1708, 1615, 1496, 1454, 1323, 1275, 1156, 1109

HRMS (DART) m/z: [M+H] + Calculated for $\mathrm{C}_{28} \mathrm{H}_{29} \mathrm{NO}_{5} \mathrm{FS} 510.1745$; Found 510.1748

MP $138-140{ }^{\circ} \mathrm{C}$

methyl 1-benzyl-3-(((2-(tert-butoxy)-2-oxoethyl)sulfonyl)methyl)-2-oxo-3-phenylindoline-5carboxylate (2k)

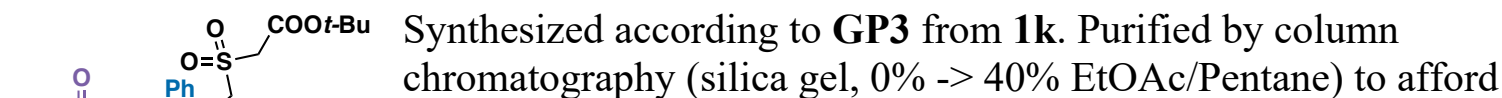
product $\mathbf{2 k}$ as a white solid ( $77 \mathrm{mg}, 70 \%$ yield).

${ }^{1} \mathbf{H}$ NMR $\left(500 \mathrm{MHz}, \mathrm{CDCl}_{3}\right) \delta 8.18-8.15(\mathrm{~m}, 1 \mathrm{H}), 8.01(\mathrm{dd}, J=8.3$, $1.7 \mathrm{~Hz}, 1 \mathrm{H}), 7.41-7.32(\mathrm{~m}, 5 \mathrm{H}), 7.31-7.27(\mathrm{~m}, 3 \mathrm{H}), 7.26-7.19(\mathrm{~m}, 2 \mathrm{H}), 6.81(\mathrm{~d}, J=8.3 \mathrm{~Hz}$, $1 \mathrm{H}), 5.09(\mathrm{~d}, J=16.0 \mathrm{~Hz}, 1 \mathrm{H}), 4.86(\mathrm{~d}, J=15.9 \mathrm{~Hz}, 1 \mathrm{H}), 4.64(\mathrm{~d}, J=15.0 \mathrm{~Hz}, 1 \mathrm{H}), 4.44(\mathrm{~d}, J=$ $14.9 \mathrm{~Hz}, 1 \mathrm{H}), 3.88(\mathrm{~s}, 4 \mathrm{H}), 3.49(\mathrm{~d}, J=14.8 \mathrm{~Hz}, 1 \mathrm{H}), 1.52(\mathrm{~s}, 9 \mathrm{H})$.

${ }^{13}$ C NMR $\left(126 \mathrm{MHz}, \mathrm{CDCl}_{3}\right) \delta 176.6,166.4,162.0,147.7,137.3,135.0,132.0,129.2,128.8$, 128.5, 128.5, 127.8, 127.2, 126.9, 126.4, 124.6, 109.8, 84.5, 60.7, 59.0, 52.8, 52.1, 44.7, 27.8. IR (ATR) 2986, 1711, 1618, 1502, 1448, 1389, 1338, 1264, 1159, 1109

HRMS (DART) m/z: [M+H] + Calculated for $\mathrm{C}_{30} \mathrm{H}_{32} \mathrm{NO}_{7} \mathrm{~S}$ 550.1894; Found 550.1892 MP $74-75^{\circ} \mathrm{C}$

\section{1-methyl-3-(((3-nitrobenzyl)sulfonyl)methyl)-3-phenylindolin-2-one (2I)}

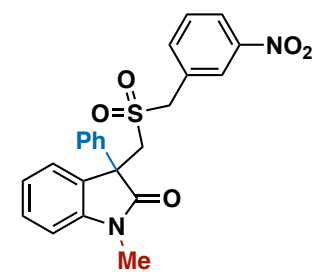

Synthesized according to GP3 from 1f. Purified by column chromatography (silica gel, $0 \%$ - $>40 \%$ EtOAc/Pentane) to afford product $2 \mathbf{l}$ as a white solid (58 mg, 66\% yield).

${ }^{1} \mathbf{H}$ NMR $\left(500 \mathrm{MHz}, \mathrm{CDCl}_{3}\right) \delta 8.22(\mathrm{dt}, J=7.4,1.8 \mathrm{~Hz}, 2 \mathrm{H}), 7.72(\mathrm{dt}, J=$ 7.6, 1.4 Hz, 1H), $7.57-7.52(\mathrm{~m}, 1 \mathrm{H}), 7.48-7.41(\mathrm{~m}, 2 \mathrm{H}), 7.41-7.36(\mathrm{~m}$, $2 \mathrm{H}), 7.34-7.29(\mathrm{~m}, 3 \mathrm{H}), 7.20(\mathrm{td}, J=7.6,1.0 \mathrm{~Hz}, 1 \mathrm{H}), 6.98(\mathrm{dt}, J=7.9,0.8 \mathrm{~Hz}, 1 \mathrm{H}), 4.28-$ 4.18 (m, 3H), 3.84 (d, $J=14.7 \mathrm{~Hz}, 1 \mathrm{H}), 3.27$ (s, 3H).

${ }^{13}$ C NMR $\left(126 \mathrm{MHz}, \mathrm{CDCl}_{3}\right) \delta 176.3,148.4,144.3,137.4,137.0,129.9,129.7,129.2,129.1$, 128.4, 128.3, 126.5, 126.0, 125.8, 124.0, 122.7, 109.3, 60.7, 59.1, 53.0, 27.0.

IR (ATR) 2923, 1711, 1621, 1531, 1502, 1473, 1359, 1318, 1264, 1113

HRMS (DART) m/z: $[\mathrm{M}+\mathrm{H}]+$ Calculated for $\mathrm{C}_{23} \mathrm{H}_{21} \mathrm{~N}_{2} \mathrm{O}_{5} \mathrm{~S} 437.1165$; Found 437.1163 MP $60-63{ }^{\circ} \mathrm{C}$ 
3-(((3-iodobenzyl)sulfonyl)methyl)-1-methyl-3-phenylindolin-2-one (2m)

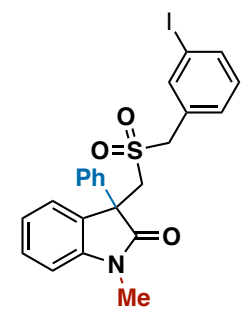

Synthesized according to GP3 from 1f. Purified by column chromatography (silica gel, $0 \%->40 \%$ EtOAc/Pentane) to afford product $\mathbf{2} \mathbf{m}$ as a white solid (68 $\mathrm{mg}, 66 \%$ yield).

${ }^{1} \mathbf{H}$ NMR $\left(500 \mathrm{MHz}, \mathrm{CDCl}_{3}\right) \delta 7.77(\mathrm{t}, J=1.7 \mathrm{~Hz}, 1 \mathrm{H}), 7.71$ (ddd, $J=8.0,1.8$, $1.0 \mathrm{~Hz}, 1 \mathrm{H}), 7.45-7.26(\mathrm{~m}, 9 \mathrm{H}), 7.20(\mathrm{td}, J=7.6,1.0 \mathrm{~Hz}, 1 \mathrm{H}), 7.10(\mathrm{t}, J=7.8$ $\mathrm{Hz}, 1 \mathrm{H}), 6.96(\mathrm{dt}, J=7.6,0.8 \mathrm{~Hz}, 1 \mathrm{H}), 4.20(\mathrm{~d}, J=14.5 \mathrm{~Hz}, 1 \mathrm{H}), 4.13(\mathrm{~d}, J=$ $13.7 \mathrm{~Hz}, 1 \mathrm{H}), 4.00(\mathrm{~d}, J=13.6 \mathrm{~Hz}, 1 \mathrm{H}), 3.75(\mathrm{dd}, J=14.5,0.6 \mathrm{~Hz}, 1 \mathrm{H}), 3.26(\mathrm{~s}, 3 \mathrm{H})$.

${ }^{13}$ C NMR $\left(126 \mathrm{MHz}, \mathrm{CDCl}_{3}\right) \delta 176.38,144.33,139.67,138.11,137.64,130.51,130.16,129.67$, 129.53, 129.02, 128.26, 126.58, 125.84, 122.62, 109.15, 94.48, 60.91, 58.39, 52.96, 26.97.

IR (ATR) 3061, 2984, 2917, 1702, 1616, 1507, 1472, 1358, 1122, 686

HRMS (DART) m/z: [M+H]+ Calculated for $\mathrm{C}_{23} \mathrm{H}_{21} \mathrm{NO}_{5} \mathrm{SI} 518.0281$; Found 518.0273

MP $154-155^{\circ} \mathrm{C}$

\section{3-((butylsulfonyl)methyl)-1-methyl-3-phenylindolin-2-one (2n)}

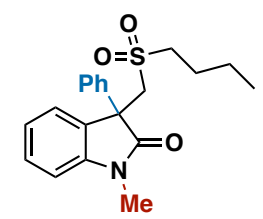

Synthesized according to GP3 from 1f. Purified by column chromatography (silica gel, $0 \%$-> 40\% EtOAc/Pentane) to afford product $\mathbf{2 n}$ as an off-white solid (23 mg, 32\% yield)

${ }^{1} \mathbf{H}$ NMR $\left(500 \mathrm{MHz}, \mathrm{CDCl}_{3}\right) \delta 7.46(\mathrm{ddd}, J=7.4,1.3,0.6 \mathrm{~Hz}, 1 \mathrm{H}), 7.44-7.37(\mathrm{~m}, 3 \mathrm{H}), 7.34-$ $7.26(\mathrm{~m}, 3 \mathrm{H}), 7.18(\mathrm{td}, J=7.6,1.0 \mathrm{~Hz}, 1 \mathrm{H}), 6.95(\mathrm{~m}, J=7.9,0.8 \mathrm{~Hz}, 1 \mathrm{H}), 4.18(\mathrm{~d}, J=14.4 \mathrm{~Hz}$, $1 \mathrm{H}), 3.89(\mathrm{~d}, J=14.4 \mathrm{~Hz}, 1 \mathrm{H}), 3.24(\mathrm{~s}, 3 \mathrm{H}), 2.78-2.65(\mathrm{~m}, 2 \mathrm{H}), 1.76-1.66(\mathrm{~m}, 2 \mathrm{H}), 1.36(\mathrm{~h}, J$ $=7.4 \mathrm{~Hz}, 2 \mathrm{H}), 0.89(\mathrm{t}, J=7.4 \mathrm{~Hz}, 3 \mathrm{H})$.

${ }^{13}$ C NMR $\left(126 \mathrm{MHz}, \mathrm{CDCl}_{3}\right) \delta 176.31,144.45,137.86,129.52,128.95,128.17,126.54,125.96$, $122.39,109.10,59.19,55.29,52.68,26.91,23.70,21.56,13.47$.

IR (ATR) 2972, 2929, 2886, 1702, 1613, 1500, 1472, 1321, 1138, 771

HRMS (DART) m/z: [M+H]+ Calculated for $\mathrm{C}_{20} \mathrm{H}_{24} \mathrm{NO}_{3} \mathrm{~S} 358.1471$; Found 358.1467

MP $132-133{ }^{\circ} \mathrm{C}$

tert-butyl 2-(((3-methyl-2,3-dihydrobenzofuran-3-yl)methyl)sulfonyl)acetate (4a)

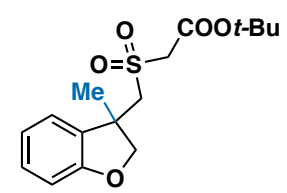

Synthesized according to GP3 from 3a. Purified by column chromatography (silica gel, $0 \%$-> 25\% DCM/Pentane, visualized with CAM stain) to afford product 4 a as a clear yellow oil (49 $\mathrm{mg}, 75 \%$ yield).

1 mmol Scale: Performed identically to GP3 from 3a except on a $1 \mathrm{mmol}$ scale. Purified by column chromatography (silica gel, $0 \%->25 \%$ DCM/Pentane, visualized with CAM stain) to yield $221 \mathrm{mg}$ of $\mathbf{4 a}$ (68\% yield) as a clear yellow oil.

${ }^{1} \mathbf{H}$ NMR $\left(500 \mathrm{MHz}, \mathrm{CDCl}_{3}\right) \delta 7.22-7.13(\mathrm{~m}, 2 \mathrm{H}), 6.91(\mathrm{td}, J=7.5,1.0 \mathrm{~Hz}, 1 \mathrm{H}), 6.84(\mathrm{dt}, J=$ 8.0, $0.8 \mathrm{~Hz}, 1 \mathrm{H}), 4.86(\mathrm{~d}, J=9.5 \mathrm{~Hz}, 1 \mathrm{H}), 4.33(\mathrm{dd}, J=9.5,0.6 \mathrm{~Hz}, 1 \mathrm{H}), 3.81-3.60(\mathrm{~m}, 4 \mathrm{H})$, $1.68(\mathrm{~s}, 3 \mathrm{H}), 1.49(\mathrm{~s}, 9 \mathrm{H})$. 
${ }^{13}$ C NMR $\left(126 \mathrm{MHz}, \mathrm{CDCl}_{3}\right) \delta 162.0,159.0,132.6,129.4,122.9,121.0,110.4,84.4,81.4,61.0$, $60.9,44.6,27.8,24.6$.

IR (ATR) 2989, 2942, 1729, 1597, 1481, 1460, 1326, 1143, 1109, 977

HRMS (DART) m/z: [M+NH4]+ Calculated for $\mathrm{C}_{16} \mathrm{H}_{26} \mathrm{NO}_{5} \mathrm{~S} 344.1526$; Found 344.1527

tert-butyl 2-(((5-chloro-3-methyl-2,3-dihydrobenzofuran-3-yl)methyl)sulfonyl)acetate (4b)

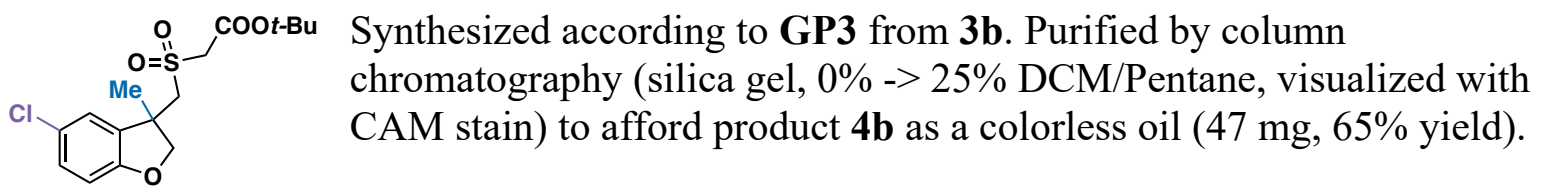

${ }^{1} \mathbf{H}$ NMR $\left(500 \mathrm{MHz}, \mathrm{CDCl}_{3}\right) \delta 7.16-7.09(\mathrm{~m}, 2 \mathrm{H}), 6.76(\mathrm{~d}, J=8.4 \mathrm{~Hz}, 1 \mathrm{H}), 4.89(\mathrm{~d}, J=9.6 \mathrm{~Hz}$ $1 \mathrm{H}), 4.36(\mathrm{dd}, J=9.7,0.7 \mathrm{~Hz}, 1 \mathrm{H}), 3.82(\mathrm{~d}, J=14.8 \mathrm{~Hz}, 1 \mathrm{H}), 3.75(\mathrm{dd}, J=14.7,0.7 \mathrm{~Hz}, 1 \mathrm{H})$, $3.69(\mathrm{~d}, J=14.1 \mathrm{~Hz}, 1 \mathrm{H}), 3.63-3.58(\mathrm{~m}, 1 \mathrm{H}), 1.67(\mathrm{~s}, 3 \mathrm{H}), 1.50(\mathrm{~s}, 9 \mathrm{H})$.

${ }^{13} \mathbf{C ~ N M R}\left(126 \mathrm{MHz}, \mathrm{CDCl}_{3}\right) \delta 162.0,157.6,134.6,129.3,125.7,123.2,111.5,84.5,81.9,61.0$, $60.6,44.8,27.8,24.6$.

IR (ATR) 2971, 2945, 1726, 1484, 1377, 1317, 1267, 1171, 1106, 977

HRMS (DART) m/z: [M+NH 4$]+$ Calculated for $\mathrm{C}_{16} \mathrm{H}_{25} \mathrm{NO}_{5} \mathrm{SCl} 378.1136$, Found 378.1131

tert-butyl 2-(((7-methoxy-3-methyl-2,3-dihydrobenzofuran-3-yl)methyl)sulfonyl)acetate (4c)

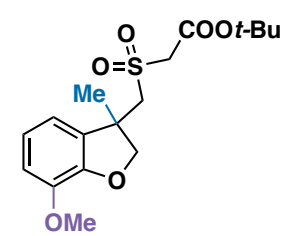

Synthesized according to GP3 from 3c. Purified by column chromatography (silica gel, $0 \%->25 \% \mathrm{DCM} /$ Pentane, visualized with CAM stain) to afford product $4 \mathbf{c}$ as a colorless oil (29 $\mathrm{mg} 41 \%$ yield).

${ }^{1} \mathbf{H}$ NMR $\left(500 \mathrm{MHz}, \mathrm{CDCl}_{3}\right) \delta 6.88(\mathrm{dd}, J=8.2,7.4 \mathrm{~Hz}, 1 \mathrm{H}), 6.78(\mathrm{~m}, 2 \mathrm{H})$, $4.91(\mathrm{~d}, J=9.6 \mathrm{~Hz}, 1 \mathrm{H}), 4.40(\mathrm{dd}, J=9.6,0.6 \mathrm{~Hz}, 1 \mathrm{H}), 3.88(\mathrm{~s}, 3 \mathrm{H}), 3.84-3.57$ (m, $4 \mathrm{H}), 1.67$ $(\mathrm{s}, 3 \mathrm{H}), 1.49(\mathrm{~s}, 9 \mathrm{H})$.

${ }^{13} \mathrm{C}$ NMR $\left(126 \mathrm{MHz}, \mathrm{CDCl}_{3}\right) \delta 162.0,147.3,145.0,133.8,121.8,114.8,112.2,84.4,82.0,61.1$, $60.8,56.0,45.2,27.8,24.5$.

IR (ATR) 2980, 1726, 1621, 1585, 1496, 1457, 1398, 1371, 1326, 1126

HRMS (DART) m/z: [M+NH4]+ Calculated for $\mathrm{C}_{17} \mathrm{H}_{28} \mathrm{NO}_{6} \mathrm{~S} 374.1632$; Found 374.1630

tert -butyl 2-(((6-chloro-4-methylisochroman-4-yl)methyl)sulfonyl)acetate (4d)

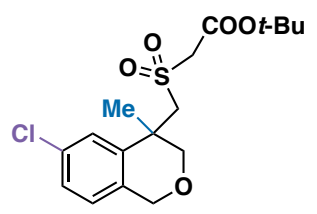

Synthesized according to GP3 from 3d. Purified by column chromatography (silica gel, $0 \%$-> 25\% DCM/Pentane, visualized with CAM stain) to afford product 4d as a colorless oil (58 $\mathrm{mg}, 77 \%$ yield).

${ }^{1} \mathbf{H}$ NMR $\left(500 \mathrm{MHz}, \mathrm{CDCl}_{3}\right) \delta 7.39(\mathrm{~d}, J=2.1 \mathrm{~Hz}, 1 \mathrm{H}), 7.19(\mathrm{dd}, J=8.2$, $2.1 \mathrm{~Hz}, 1 \mathrm{H}), 6.93(\mathrm{dd}, J=8.2,1.0 \mathrm{~Hz}, 1 \mathrm{H}), 4.83(\mathrm{~d}, J=15.2 \mathrm{~Hz}, 1 \mathrm{H}), 4.76(\mathrm{dd}, J=15.2,1.0 \mathrm{~Hz}$, $1 \mathrm{H}), 4.41(\mathrm{~d}, J=11.8 \mathrm{~Hz}, 1 \mathrm{H}), 3.84-3.71(\mathrm{~m}, 3 \mathrm{H}), 3.60-3.49(\mathrm{~m}, 2 \mathrm{H}), 1.58(\mathrm{~s}, 3 \mathrm{H}), 1.49(\mathrm{~s}$, 9H).

${ }^{13}$ C NMR (126 MHz, $\left.\mathrm{CDCl}_{3}\right) \delta 162.0,141.1,132.8,132.1,127.5,126.4,126.0,84.2,72.3,68.2$, 61.3, 60.2, 37.3, 27.8, 22.0. 
IR (ATR) 2980, 1729, 1597, 1499, 1463, 1404, 1368, 1320, 1135, 1109

HRMS (DART) m/z: [M+NH$]+$ Calculated for $\mathrm{C}_{17} \mathrm{H}_{27} \mathrm{NO}_{5} \mathrm{SCl} 392.1293$; Found 392.1290

tert-butyl 2-(((1-methyl-2,3-dihydro-1H-inden-1-yl)methyl)sulfonyl)acetate (4e)

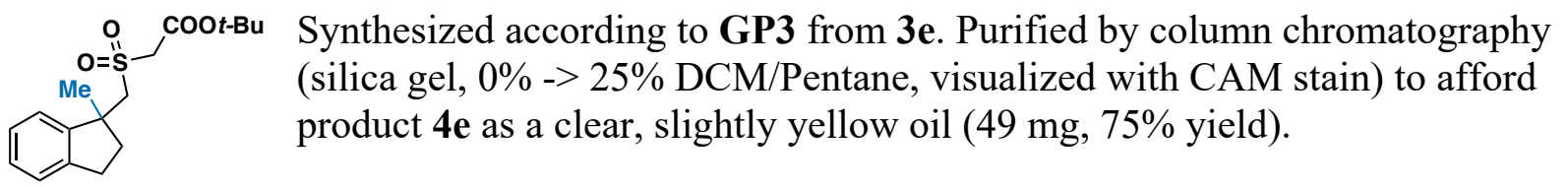

${ }^{1}$ H NMR $\left(500 \mathrm{MHz}, \mathrm{CDCl}_{3}\right) \delta 7.26-7.18(\mathrm{~m}, 4 \mathrm{H}), 3.68-3.54(\mathrm{~m}, 3 \mathrm{H}), 3.48(\mathrm{~d}, J=14.3 \mathrm{~Hz}$, 1H), $3.07-2.89$ (m, 2H), 2.57 (ddd, $J=13.1,8.1,4.9 \mathrm{~Hz}, 1 \mathrm{H}), 2.09$ (ddd, $J=13.2,8.5,7.3 \mathrm{~Hz}$, $1 \mathrm{H}), 1.60(\mathrm{~s}, 3 \mathrm{H}), 1.48(\mathrm{~s}, 9 \mathrm{H})$.

${ }^{13}$ C NMR $\left(126 \mathrm{MHz}_{\mathrm{CDCl}}\right) \delta 162.3,148.2,143.0,127.6,126.7,125.1,122.8,83.9,61.8,60.7$, 46.7, 38.6, 30.2, 27.8, 26.3.

IR (ATR) 2977, 2939, 1729,1481, 1448, 1404, 1374, 1323, 1153, 1103

HRMS (DART) m/z: [M+NH4]+ Calculated for $\mathrm{C}_{17} \mathrm{H}_{28} \mathrm{NO}_{4} \mathrm{~S} 342.1734$; Found 342.1736

diethyl 4-(((2-(tert-butoxy)-2-oxoethyl)sulfonyl)methyl)-4-methyl-3,4-dihydronaphthalene2,2(1H)-dicarboxylate $(4 \mathrm{f})$

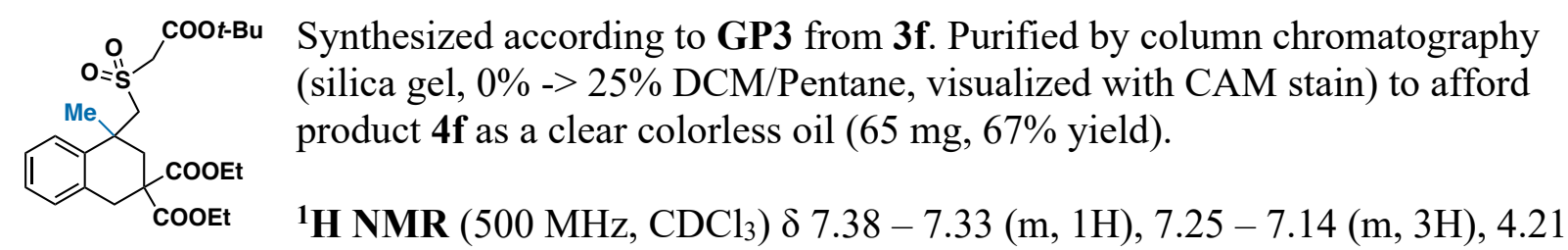

$(\mathrm{q}, J=7.1 \mathrm{~Hz}, 2 \mathrm{H}), 4.15-3.98(\mathrm{~m}, 2 \mathrm{H}), 3.69(\mathrm{~d}, J=15.0 \mathrm{~Hz}, 1 \mathrm{H}), 3.62(\mathrm{~d}, J=15.0 \mathrm{~Hz}, 1 \mathrm{H})$, $3.39-3.20(\mathrm{~m}, 4 \mathrm{H}), 2.99(\mathrm{~d}, J=14.7 \mathrm{~Hz}, 1 \mathrm{H}), 2.46(\mathrm{dd}, J=14.7,1.6 \mathrm{~Hz}, 1 \mathrm{H}), 1.61(\mathrm{~s}, 3 \mathrm{H}), 1.46$ $(\mathrm{s}, 9 \mathrm{H}), 1.26(\mathrm{t}, J=7.1 \mathrm{~Hz}, 3 \mathrm{H}), 1.14(\mathrm{t}, J=7.1 \mathrm{~Hz}, 3 \mathrm{H})$.

${ }^{13}$ C NMR $\left(126 \mathrm{MHz} \mathrm{CDCl}_{3}\right) \delta 171.3,162.3,138.7,134.6,129.4,127.4,127.0,126.5,83.9$, 63.5, 61.6, 60.1, 53.0, 40.0, 37.4, 34.8, 29.9, 27.8, 13.9.

IR (ATR) 2989, 2939, 1729, 1496, 1454, 1401, 1368, 1329, 1100, 1052

HRMS (DART) m/z: [M+NH 4$]+$ Calculated for $\mathrm{C}_{24} \mathrm{H}_{38} \mathrm{NO}_{8} \mathrm{~S} 500.2313$; Found 500.2312

\section{1-(((3-methyl-2,3-dihydrobenzofuran-3-yl)methyl)sulfonyl)piperidine (5a)}

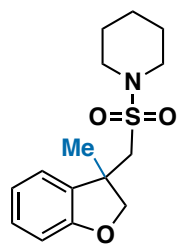

Synthesized according to GP4 from 3a. Purified by column chromatography (silica gel, $0 \%->40 \%$ EtOAc/Pentane, visualized with CAM stain) to afford product 5a as a tan oil (31 mg, 52\% yield).

${ }^{1} \mathbf{H}$ NMR $\left(500 \mathrm{MHz}, \mathrm{CDCl}_{3}\right) \delta 7.20-7.15(\mathrm{~m}, 1 \mathrm{H}), 7.11(\mathrm{dd}, J=7.4,1.4 \mathrm{~Hz}, 1 \mathrm{H})$, $6.91(\mathrm{td}, J=7.4,1.0 \mathrm{~Hz}, 1 \mathrm{H}), 6.83(\mathrm{dt}, J=8.0,0.7 \mathrm{~Hz}, 1 \mathrm{H}), 4.88(\mathrm{~d}, J=9.5 \mathrm{~Hz}, 1 \mathrm{H})$, $4.30(\mathrm{dd}, J=9.5,0.7 \mathrm{~Hz}, 1 \mathrm{H}), 3.21-3.06(\mathrm{~m}, 6 \mathrm{H}), 1.65(\mathrm{~m}, 7 \mathrm{H}), 1.57-1.50(\mathrm{~m}, 2 \mathrm{H})$.

${ }^{13} \mathrm{C}$ NMR $\left(126 \mathrm{MHz}, \mathrm{CDCl}_{3}\right) \delta 158.9,133.8,129.1,122.6,120.9,110.3,81.5,55.7,46.6,44.3$, 25.5, 24.2, 23.7.

IR (ATR) 2986, 1729, 1597, 1484, 1460, 1332, 1273, 1225, 1162, 928

HRMS (DART) m/z: [M+H]+ Calculated for $\mathrm{C}_{15} \mathrm{H}_{22} \mathrm{NO}_{3} \mathrm{~S} 296.1315$; Found 296.1315 


\section{1,3-dimethyl-3-((piperidin-1-ylsulfonyl)methyl)indolin-2-one (5b)}

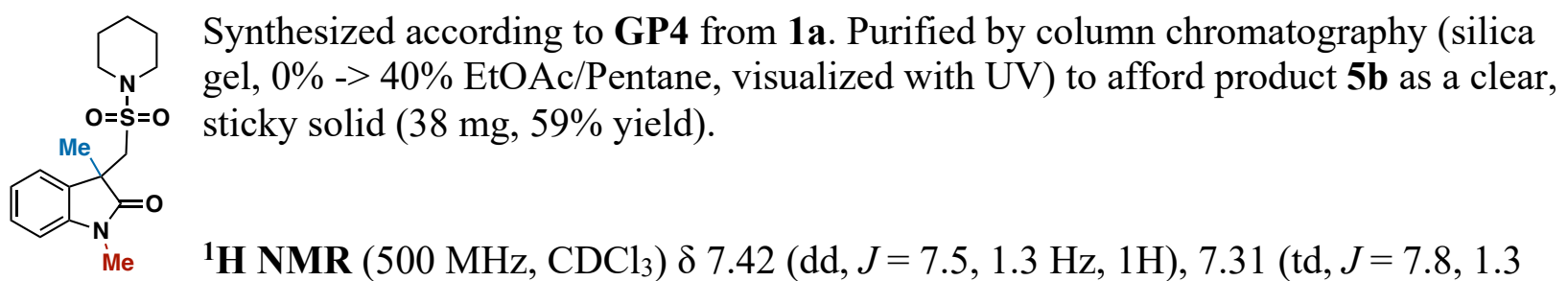
$\mathrm{Hz}, 1 \mathrm{H}), 7.11(\mathrm{td}, J=7.5,1.1 \mathrm{~Hz}, 1 \mathrm{H}), 6.87(\mathrm{~d}, J=7.8 \mathrm{~Hz}, 1 \mathrm{H}), 3.50(\mathrm{~d}, J=14.1 \mathrm{~Hz}, 1 \mathrm{H}), 3.44-$ $3.31(\mathrm{~m}, 1 \mathrm{H}), 3.25(\mathrm{~s}, 3 \mathrm{H}), 3.19-2.91(\mathrm{~m}, 4 \mathrm{H}), 1.66-1.46(\mathrm{~m}, 6 \mathrm{H}), 1.44(\mathrm{~s}, 3 \mathrm{H})$.

${ }^{13} \mathbf{C}$ NMR $\left(126 \mathrm{MHz}, \mathrm{CDCl}_{3}\right) \delta 178.3,143.1,130.6,128.6,124.4,122.6,108.4,54.8,46.3,45.7$, 41.5, 26.6, 25.5, 24.9, 23.7, 14.5.

IR (ATR) 2933, 2858, 1708, 1615, 1502, 1475, 1445, 1383, 1329, 936

HRMS (DART) m/z: [M+H]+ Calculated for $\mathrm{C}_{16} \mathrm{H}_{23} \mathrm{~N}_{2} \mathrm{O}_{3} \mathrm{~S} 323.1424$; Found 323.1423

MP $27-30{ }^{\circ} \mathrm{C}$

\section{(3-methyl-2,3-dihydrobenzofuran-3-yl)methanesulfonyl fluoride (5c)}

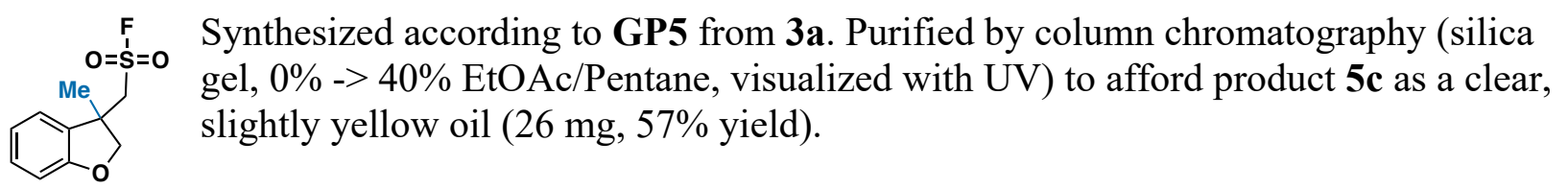

${ }^{1} \mathbf{H}$ NMR $\left(500 \mathrm{MHz}, \mathrm{CDCl}_{3}\right) \delta 7.23(\mathrm{ddd}, J=8.1,7.4,1.3 \mathrm{~Hz}, 1 \mathrm{H}), 7.15(\mathrm{ddd}, J=7.5,1.4,0.5$ $\mathrm{Hz}, 1 \mathrm{H}), 6.95(\mathrm{td}, J=7.5,1.0 \mathrm{~Hz}, 1 \mathrm{H}), 6.87(\mathrm{dt}, J=8.1,0.8 \mathrm{~Hz}, 1 \mathrm{H}), 4.79(\mathrm{~d}, J=9.7 \mathrm{~Hz}, 1 \mathrm{H})$, $4.29(\mathrm{dd}, J=9.6,0.7 \mathrm{~Hz}, 1 \mathrm{H}), 3.73-3.64(\mathrm{~m}, 2 \mathrm{H}), 1.67$ (d, $J=1.6 \mathrm{~Hz}, 3 \mathrm{H})$.

${ }^{13}$ C NMR $\left(126 \mathrm{MHz}, \mathrm{CDCl}_{3}\right) \delta 158.9,131.3,129.9,122.6,121.4,110.7,80.7,59.1(\mathrm{~d}, J=11.34$ $\mathrm{Hz}), 44.1,23.5$.

${ }^{19}$ F NMR $\left(400 \mathrm{MHz}, \mathrm{CDCl}_{3}\right) \delta 64.93$

IR (ATR) 2917, 2924, 2849, 1600, 1460, 1481, 1335, 1225, 1162, 936

HRMS (DART) m/z: [M+NH $\left.\mathrm{NH}_{4}\right]$ Calculated for $\mathrm{C}_{10} \mathrm{H}_{15} \mathrm{NO}_{3} \mathrm{FS} 248.0751$; Found 248.0756

\section{(1,3-dimethyl-2-oxoindolin-3-yl)methanesulfonyl fluoride (5d)}

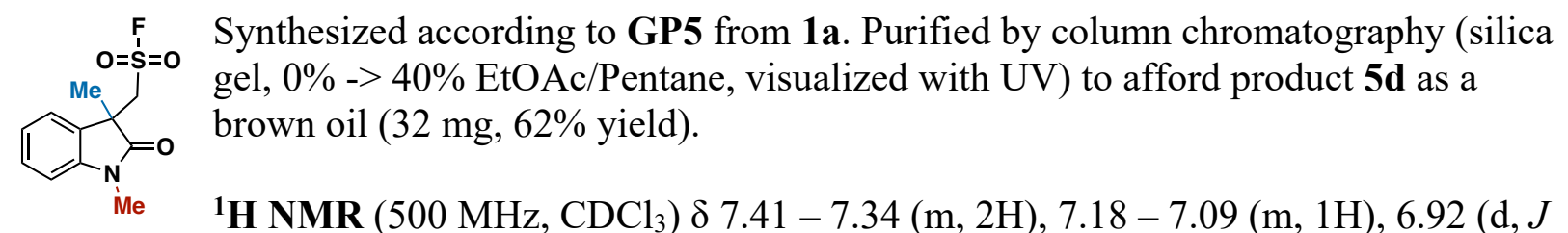
$=8.0 \mathrm{~Hz}, 1 \mathrm{H}), 4.02(\mathrm{dd}, J=14.8,7.0 \mathrm{~Hz}, 1 \mathrm{H}), 3.89(\mathrm{~d}, J=14.8 \mathrm{~Hz}, 1 \mathrm{H}), 3.26(\mathrm{~s}, 3 \mathrm{H}), 1.51(\mathrm{~s}$, $3 \mathrm{H})$.

${ }^{13}$ C NMR (126 MHz, $\left.\mathrm{CDCl}_{3}\right) \delta 176.7,143.1,129.5,128.7,123.9,123.0,109.0,56.9,45.3,26.7$, 24.3.

${ }^{19}$ F NMR $\left(400 \mathrm{MHz}, \mathrm{CDCl}_{3}\right) \delta 202.81(\mathrm{~d}, J=6.9 \mathrm{~Hz})$

IR (ATR) 2980, 1702, 1615, 1502, 1478, 1448, 1410, 1386, 1350, 1201

HRMS (DART) m/z: [M+H]+ Calculated for $\mathrm{C}_{11} \mathrm{H}_{13} \mathrm{NO}_{3} \mathrm{FS}$ 258.0595; Found 258.0598 

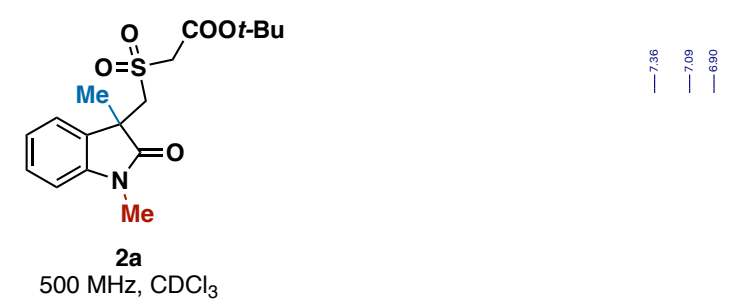

î

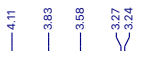

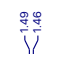

$1 / 1$

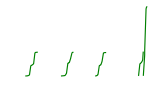

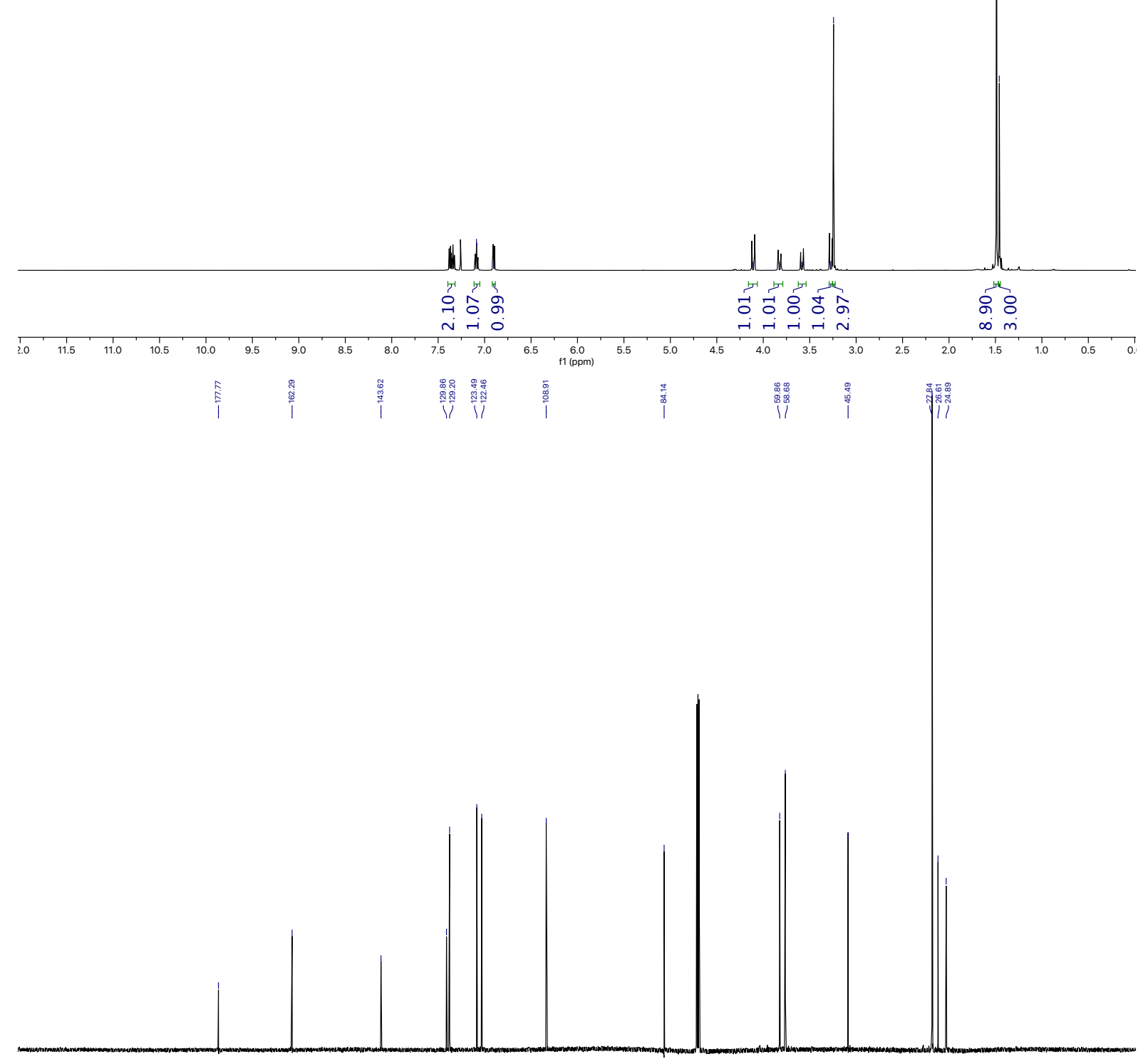

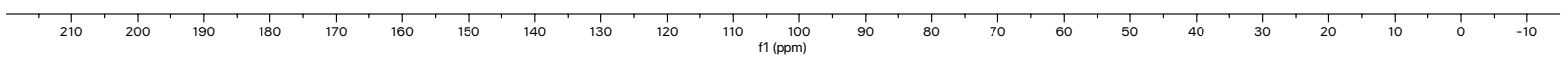



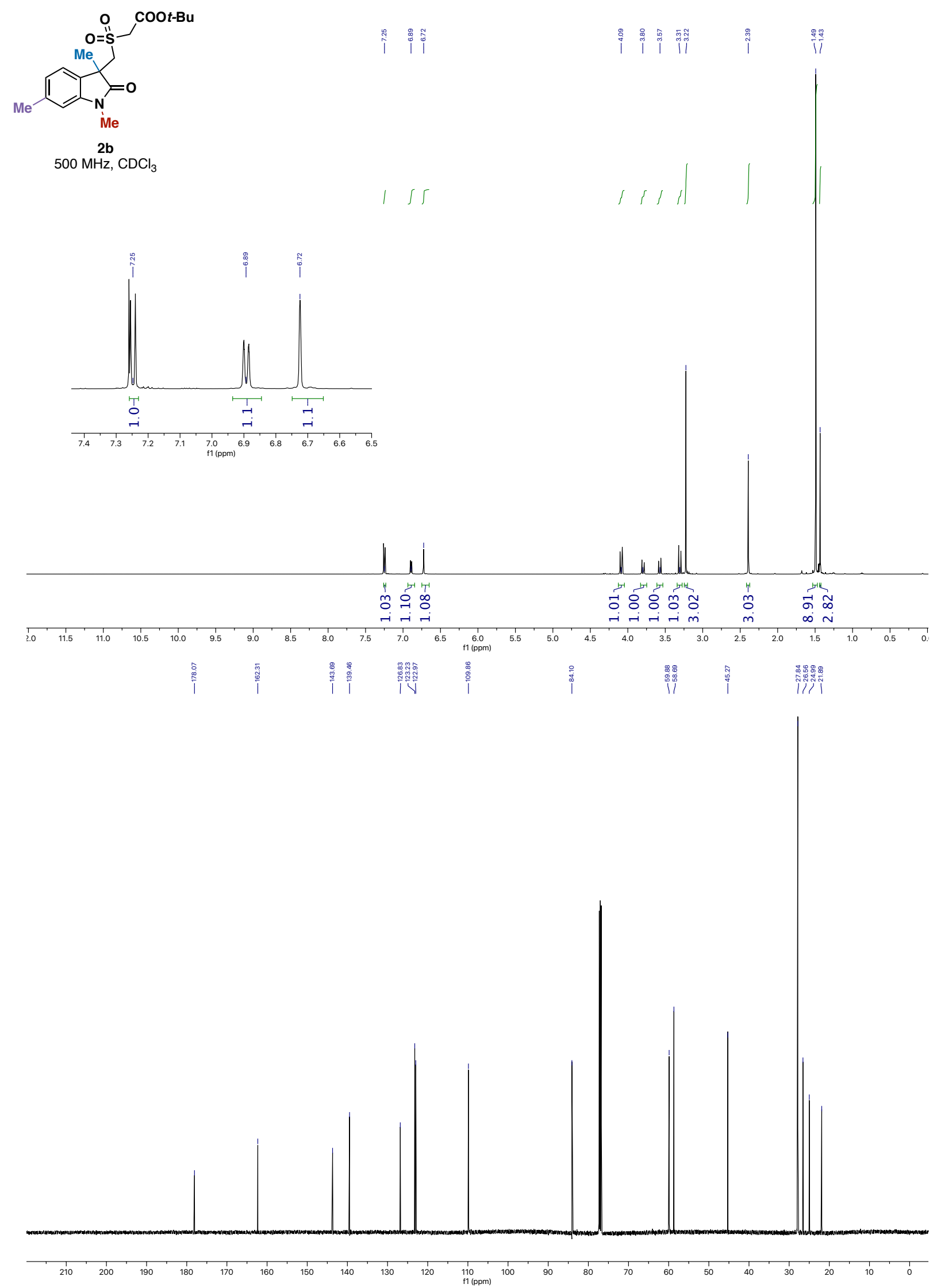

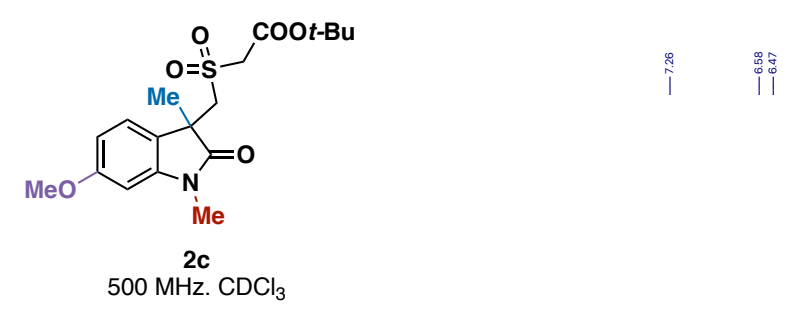

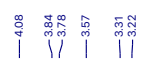

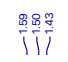
$500 \mathrm{MHz} . \mathrm{CDCl}_{3}$
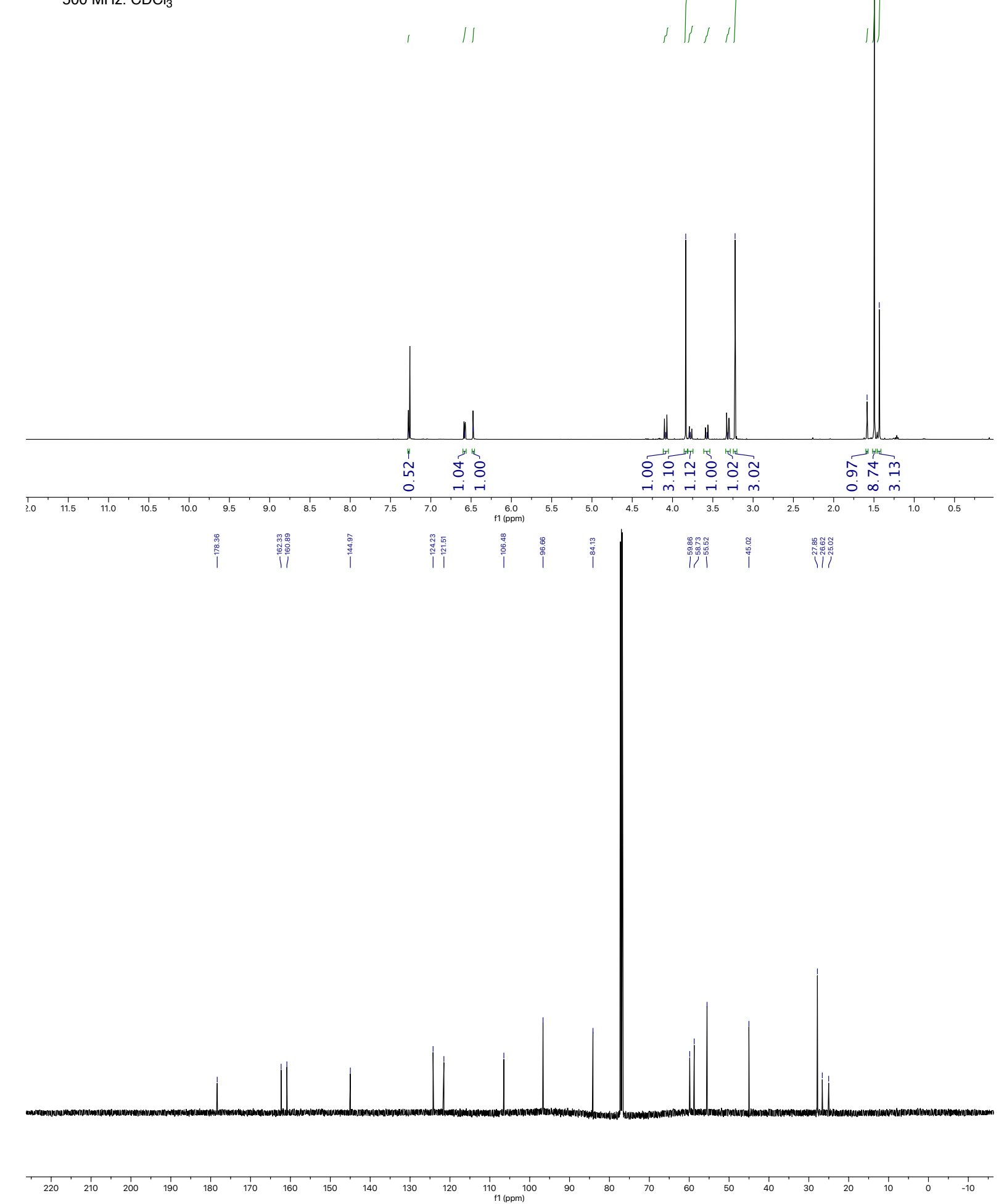


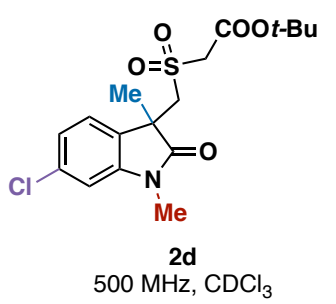

$\iint 5$

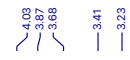

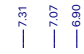

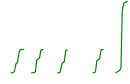

HuLlL

出

००००

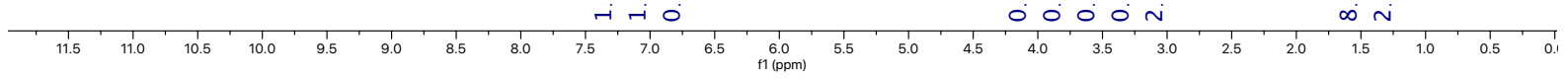

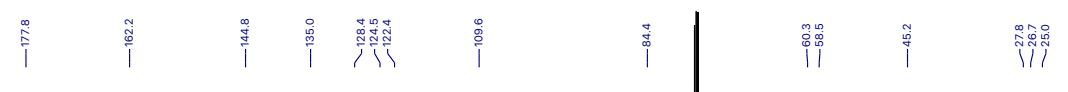

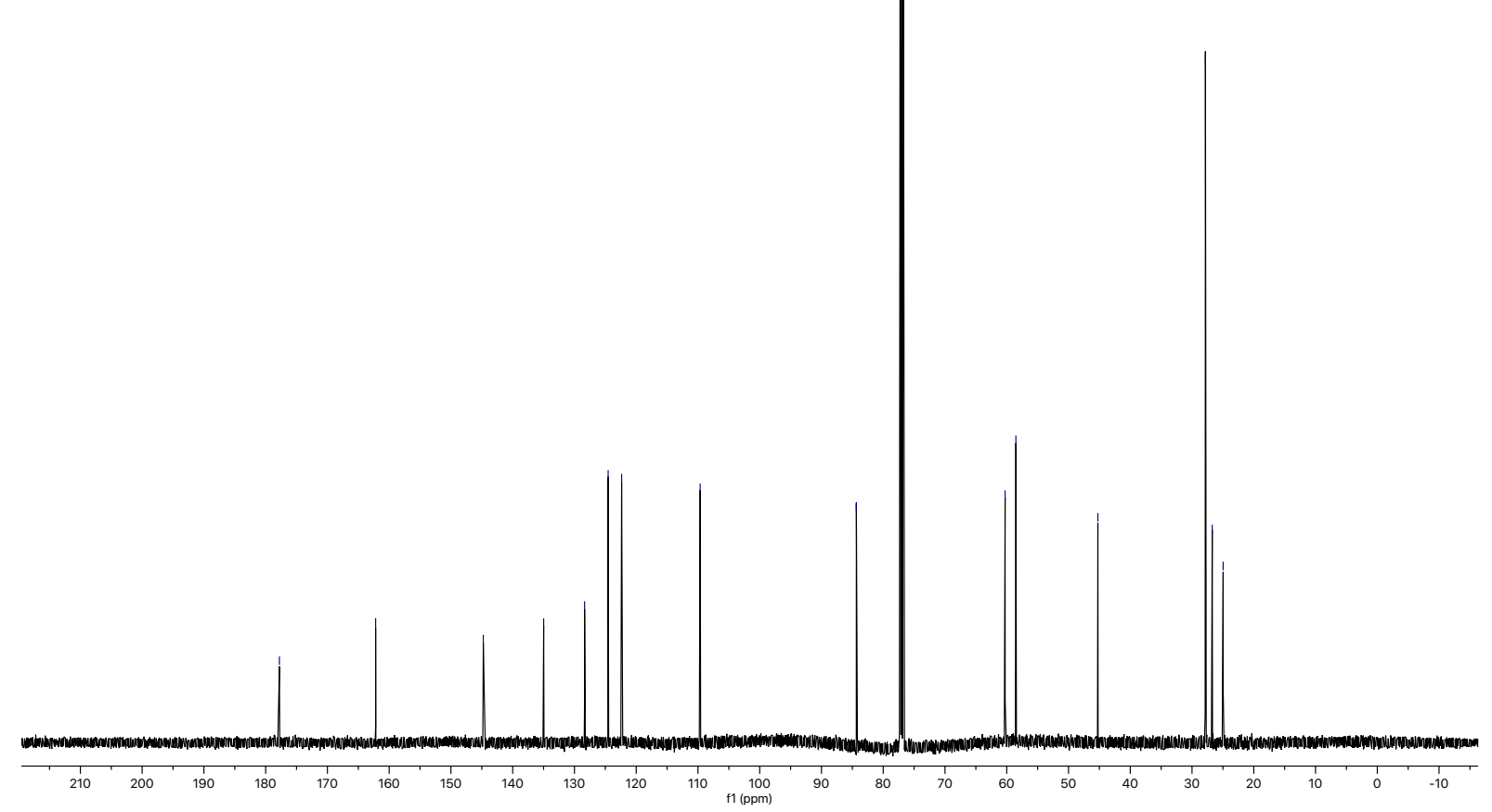




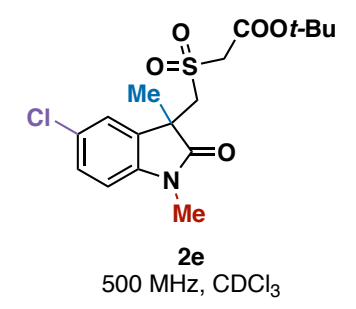

II 1

Ine

난 잉

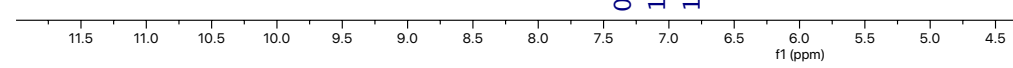

हn

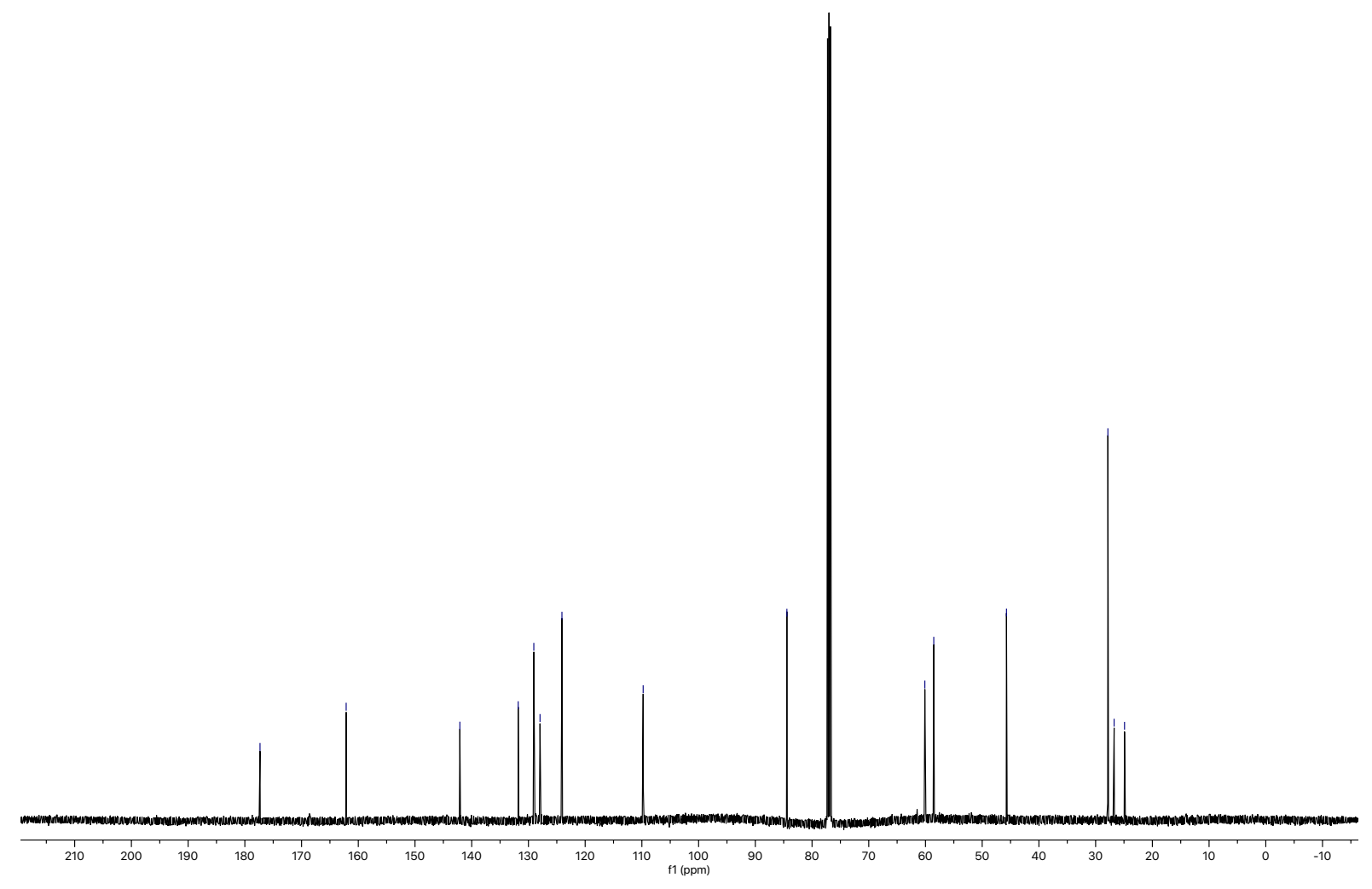




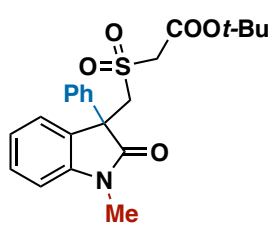

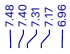

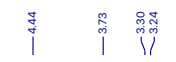

$2 f$

$500 \mathrm{MHz}, \mathrm{CDCl}_{3}$
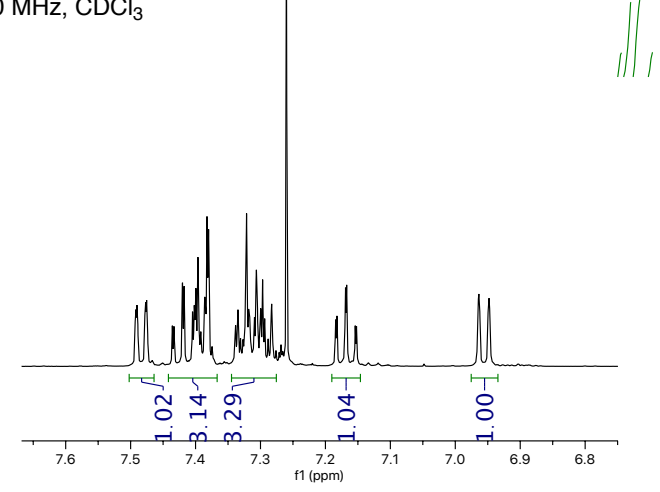

$\int 1$

M) M

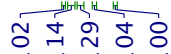

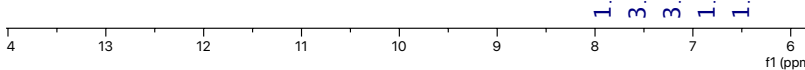

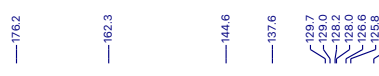

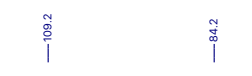

ำ

踝

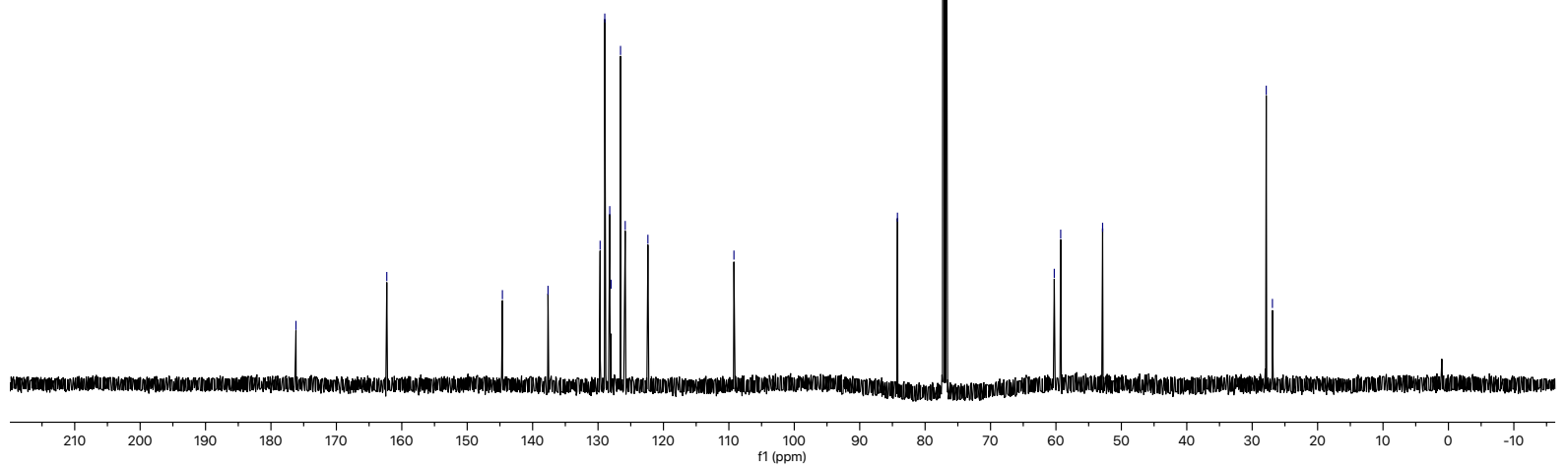



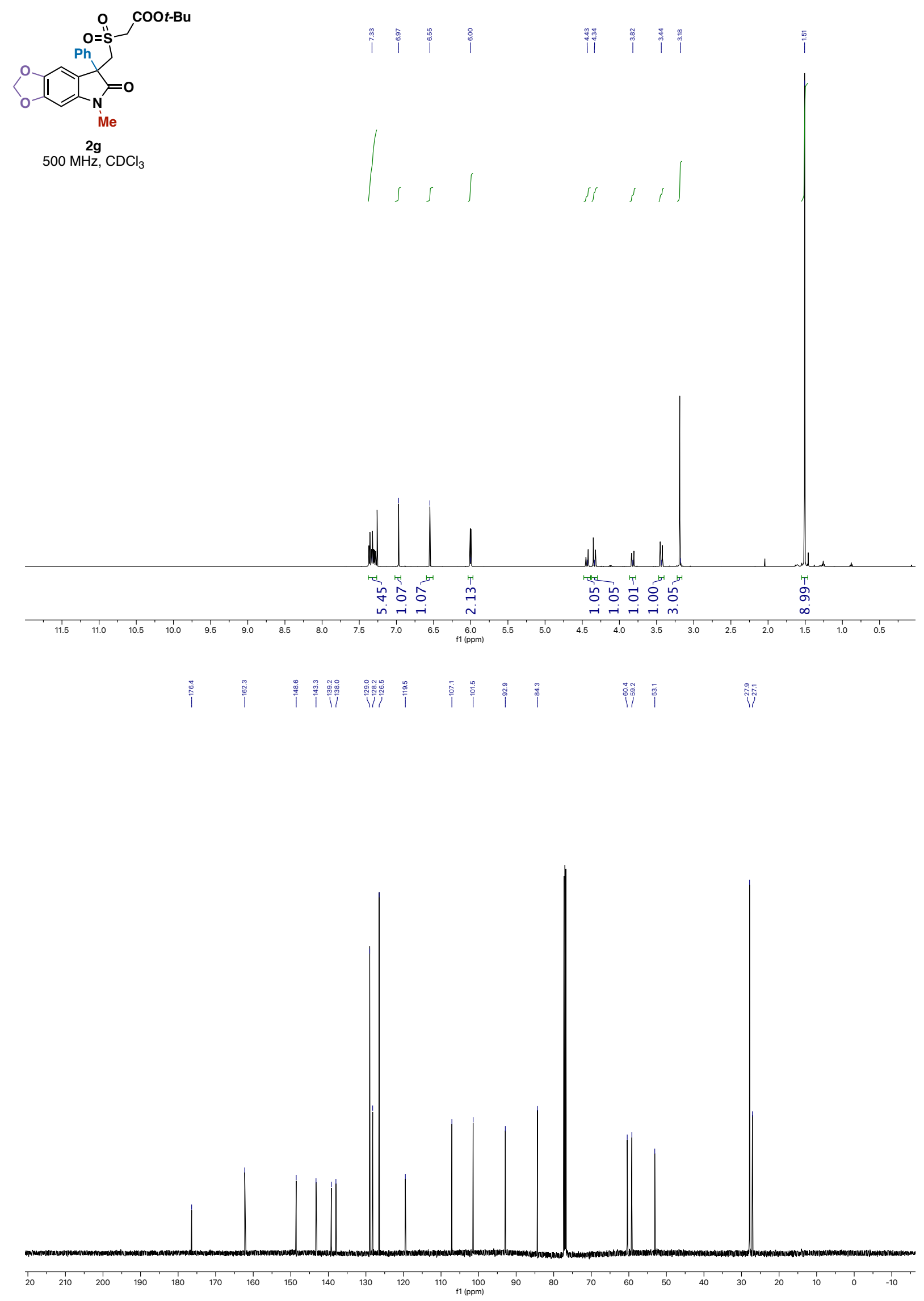

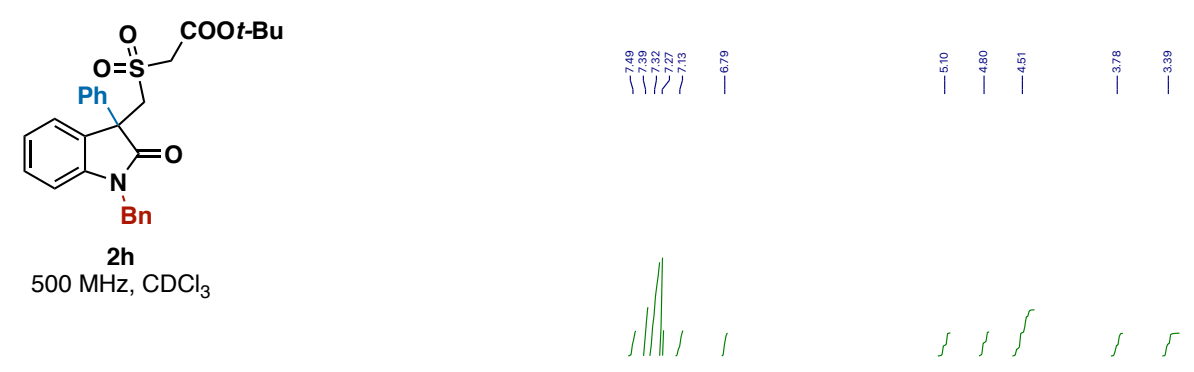

$\stackrel{2 h}{2 h} \mathrm{MHz}_{3}$
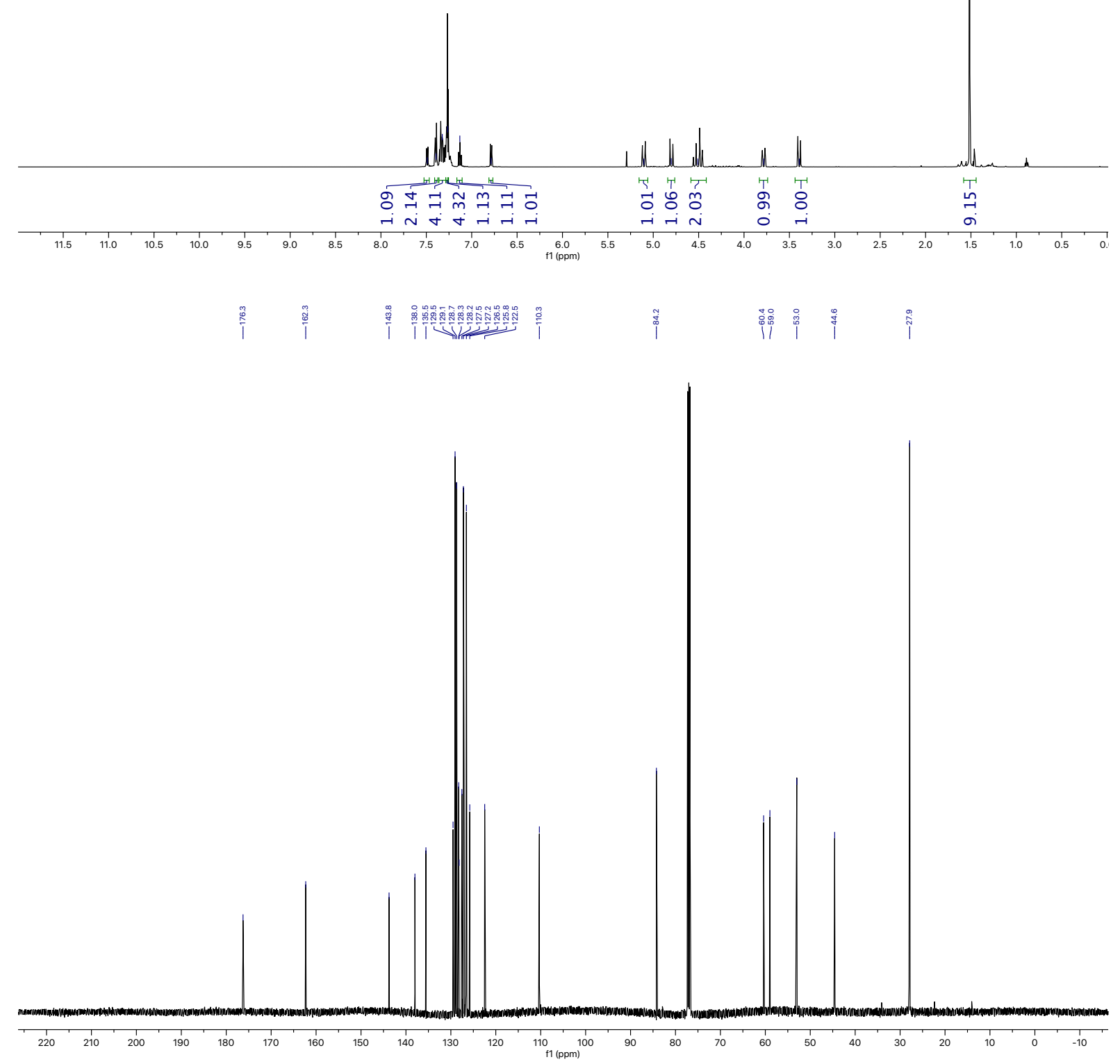

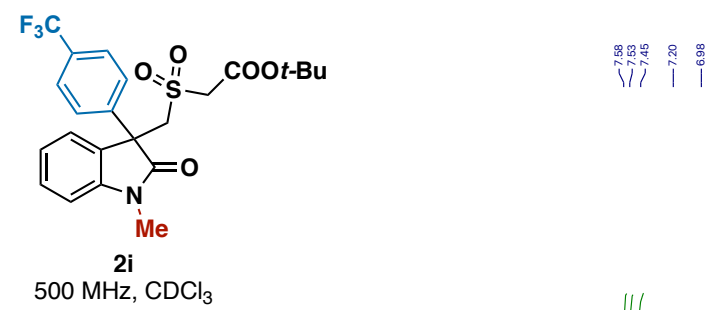

Mhe

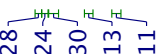

N N N

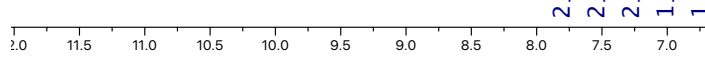

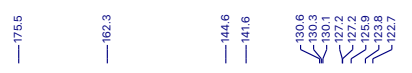

$\stackrel{\substack{0 \\:}}{i}$

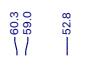

$\stackrel{\substack{\infty \\ N}}{\substack{N \\ N}}$

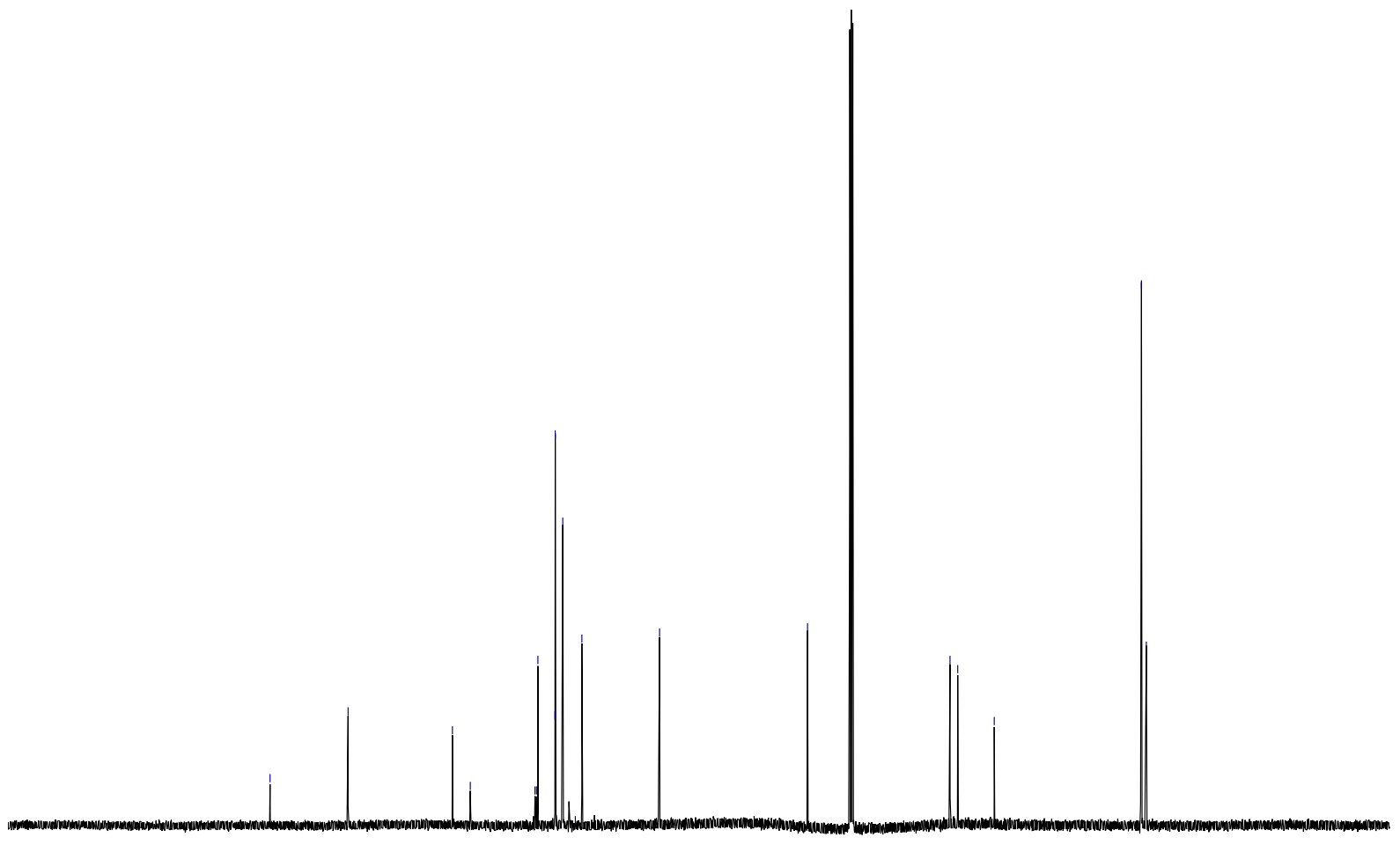

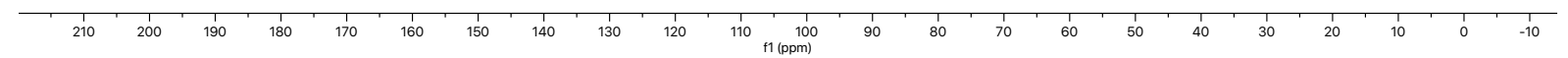


${ }^{19} \mathrm{~F} \mathrm{NMR}\left(400 \mathrm{MHz}, \mathrm{CDCl}_{3}\right)$

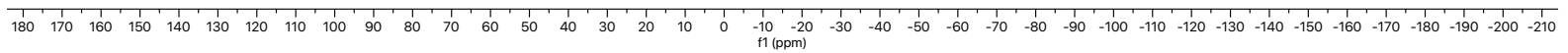



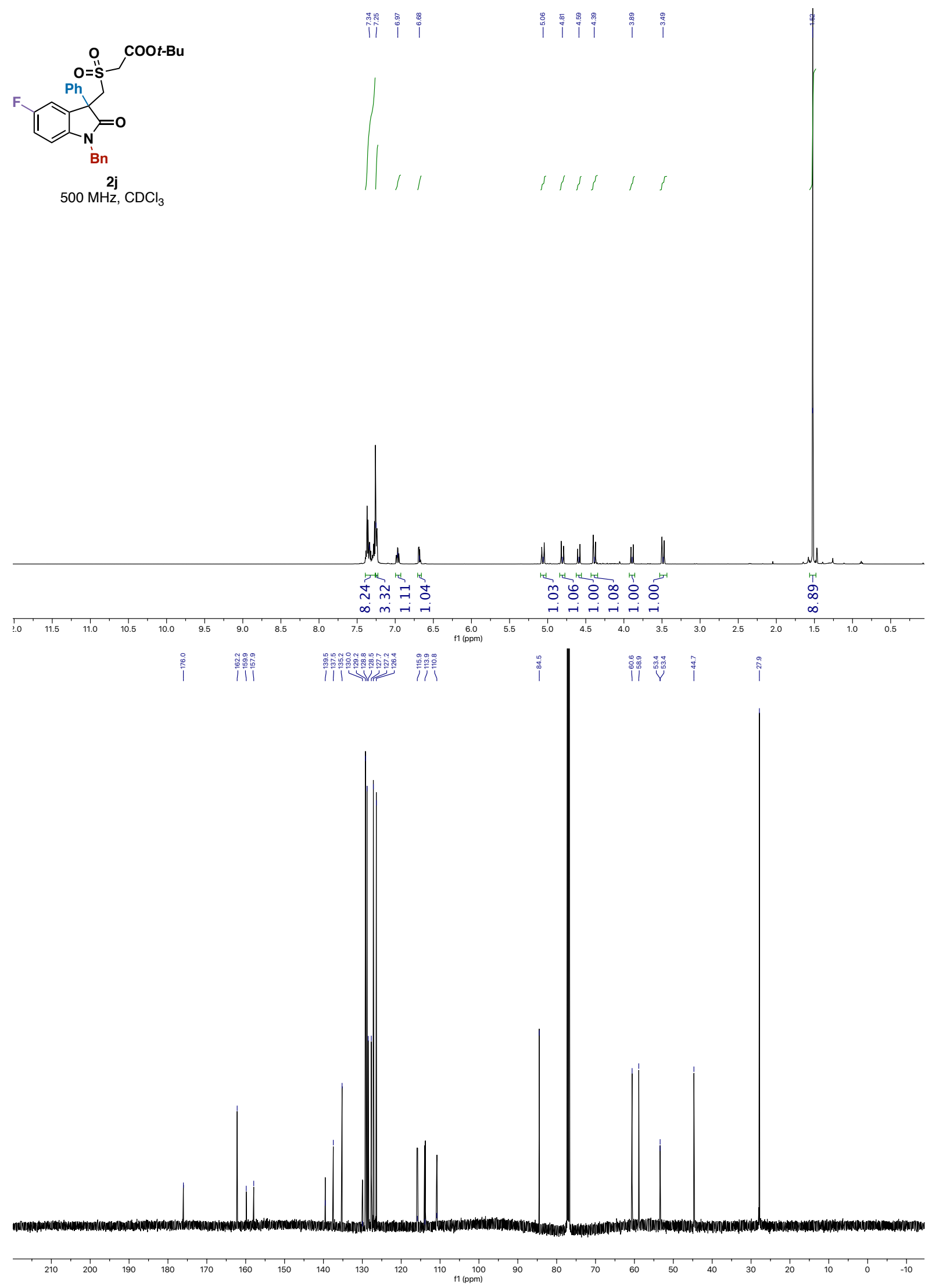
${ }^{19} \mathrm{~F} \mathrm{NMR}\left(400 \mathrm{MHz}, \mathrm{CDCl}_{3}\right)$

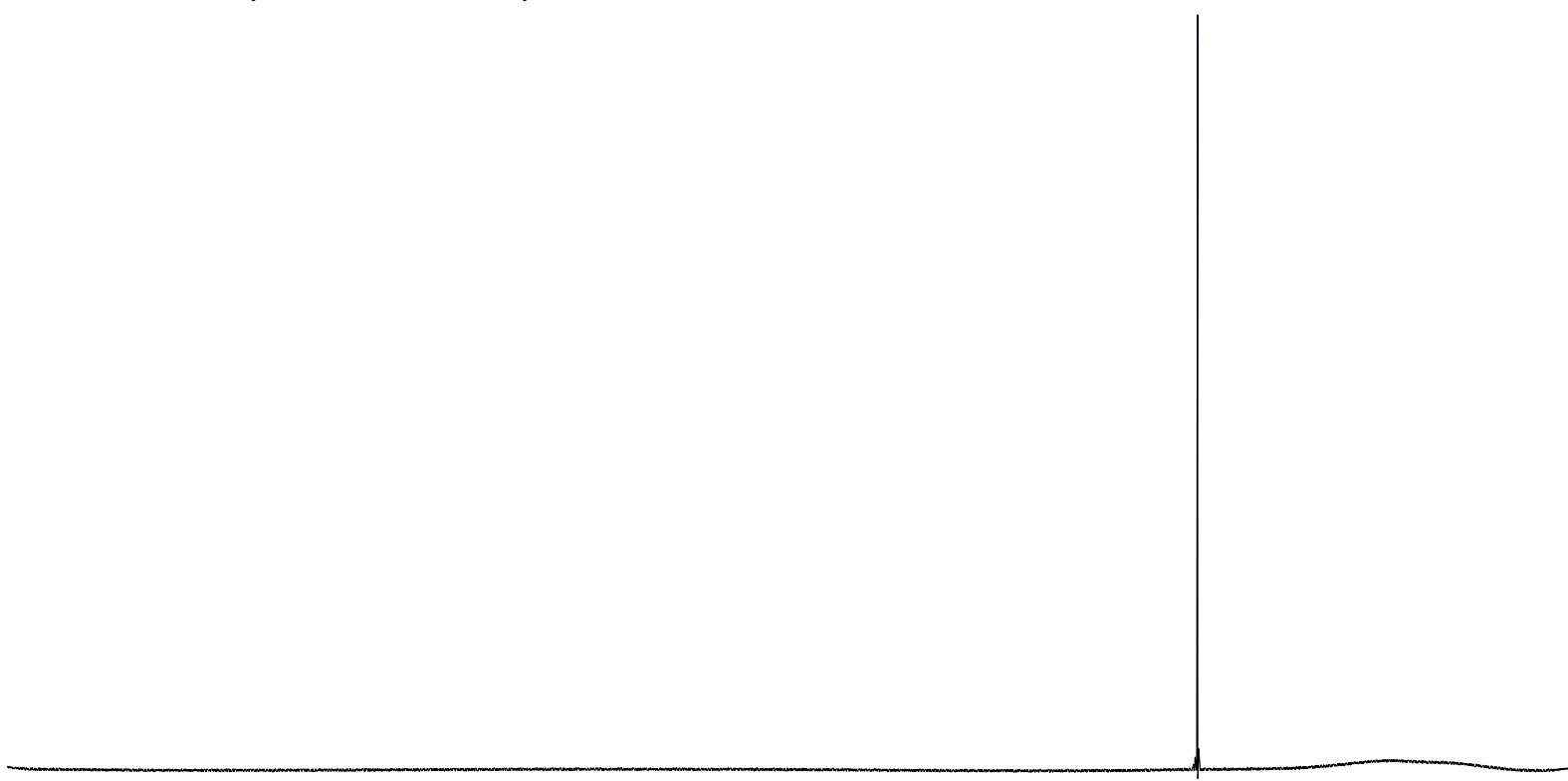

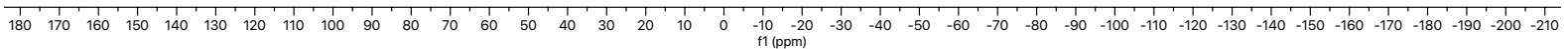




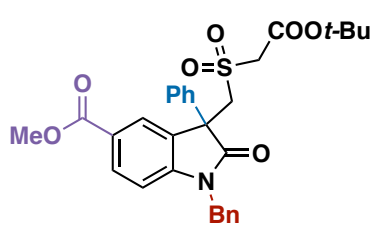

2k $500 \mathrm{MHz}, \mathrm{CDCl}_{3}$
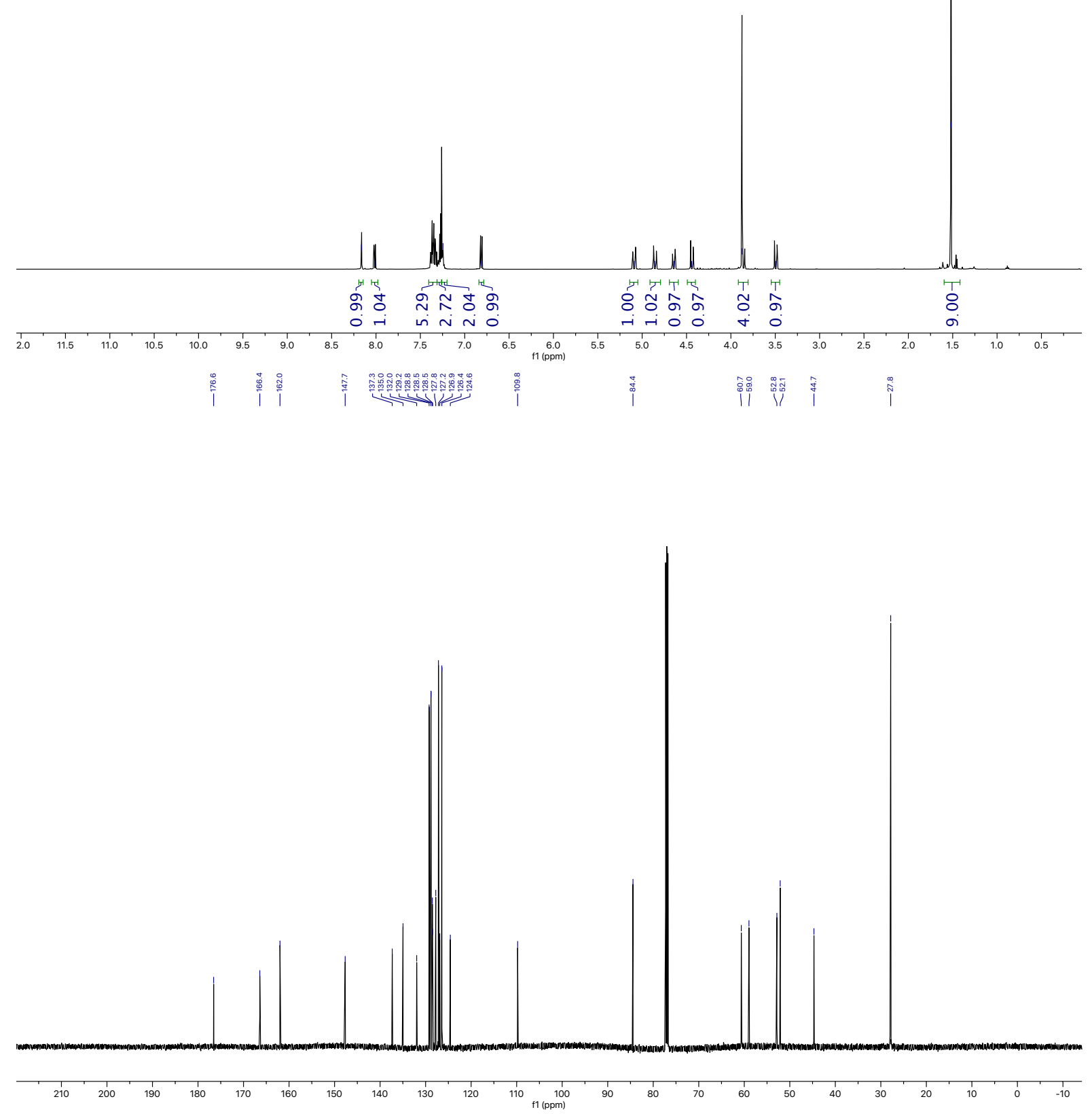


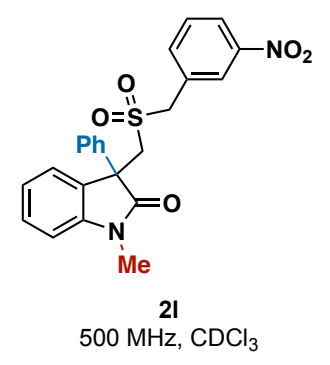

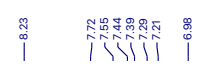

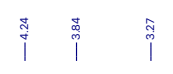
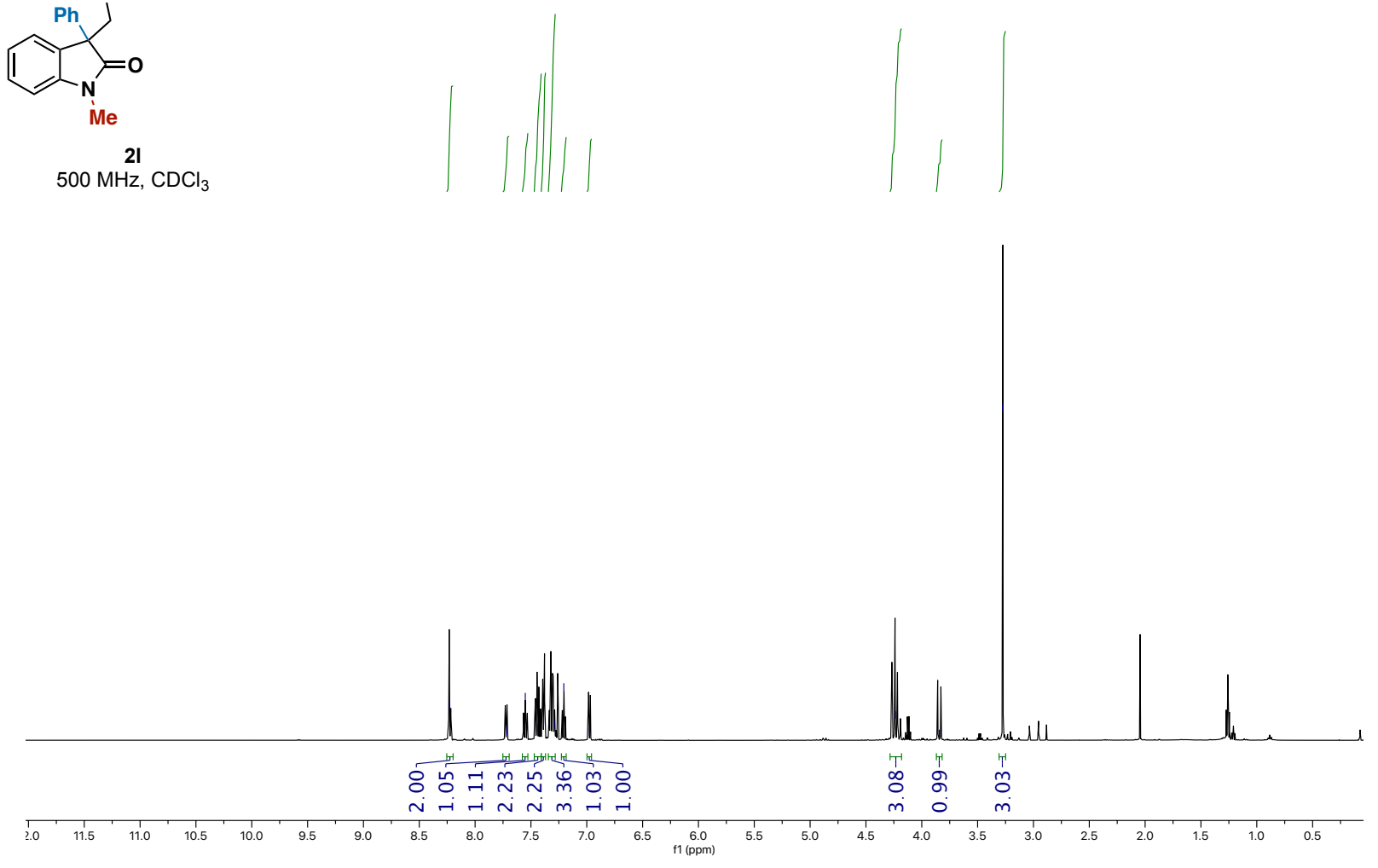

I.

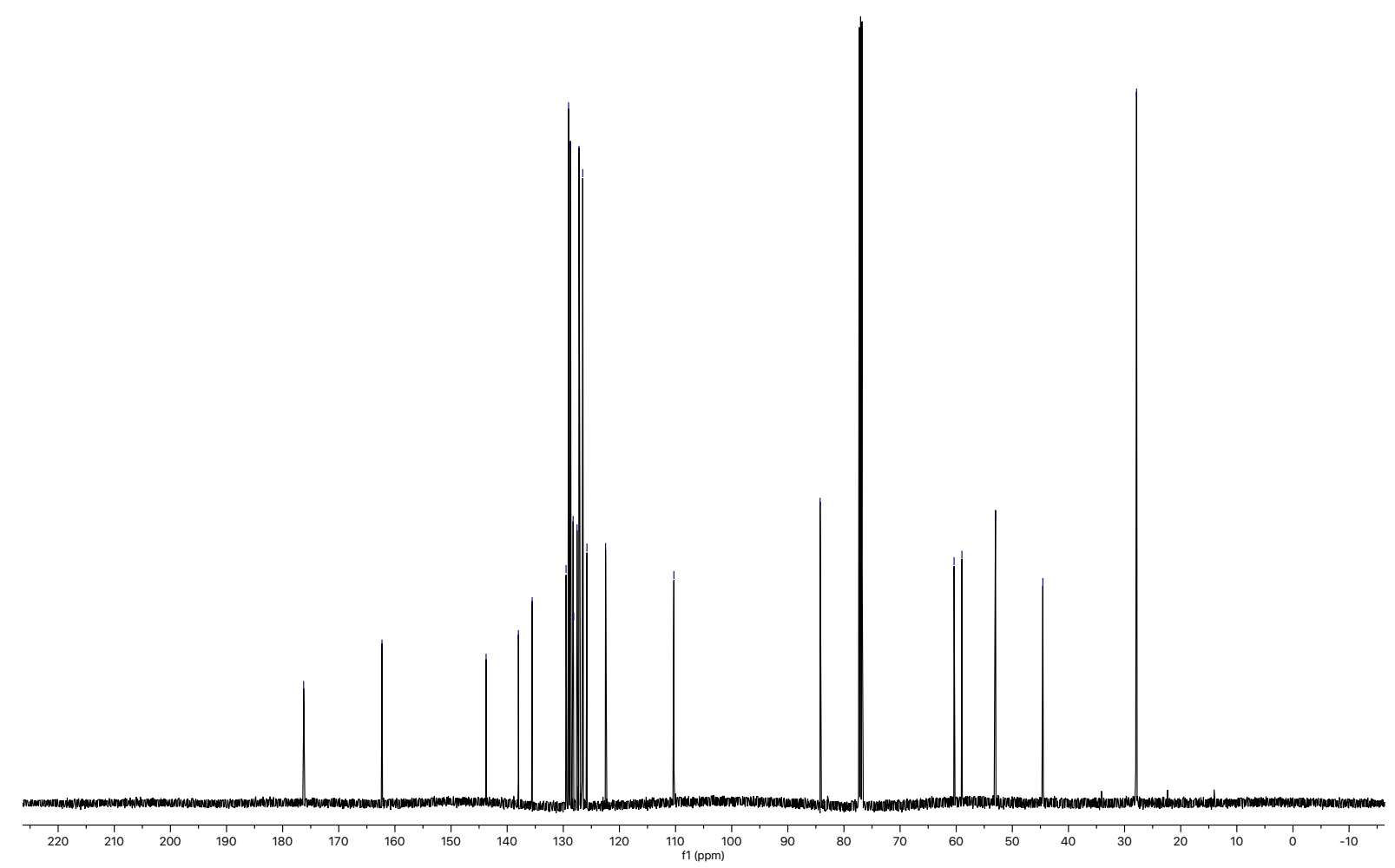



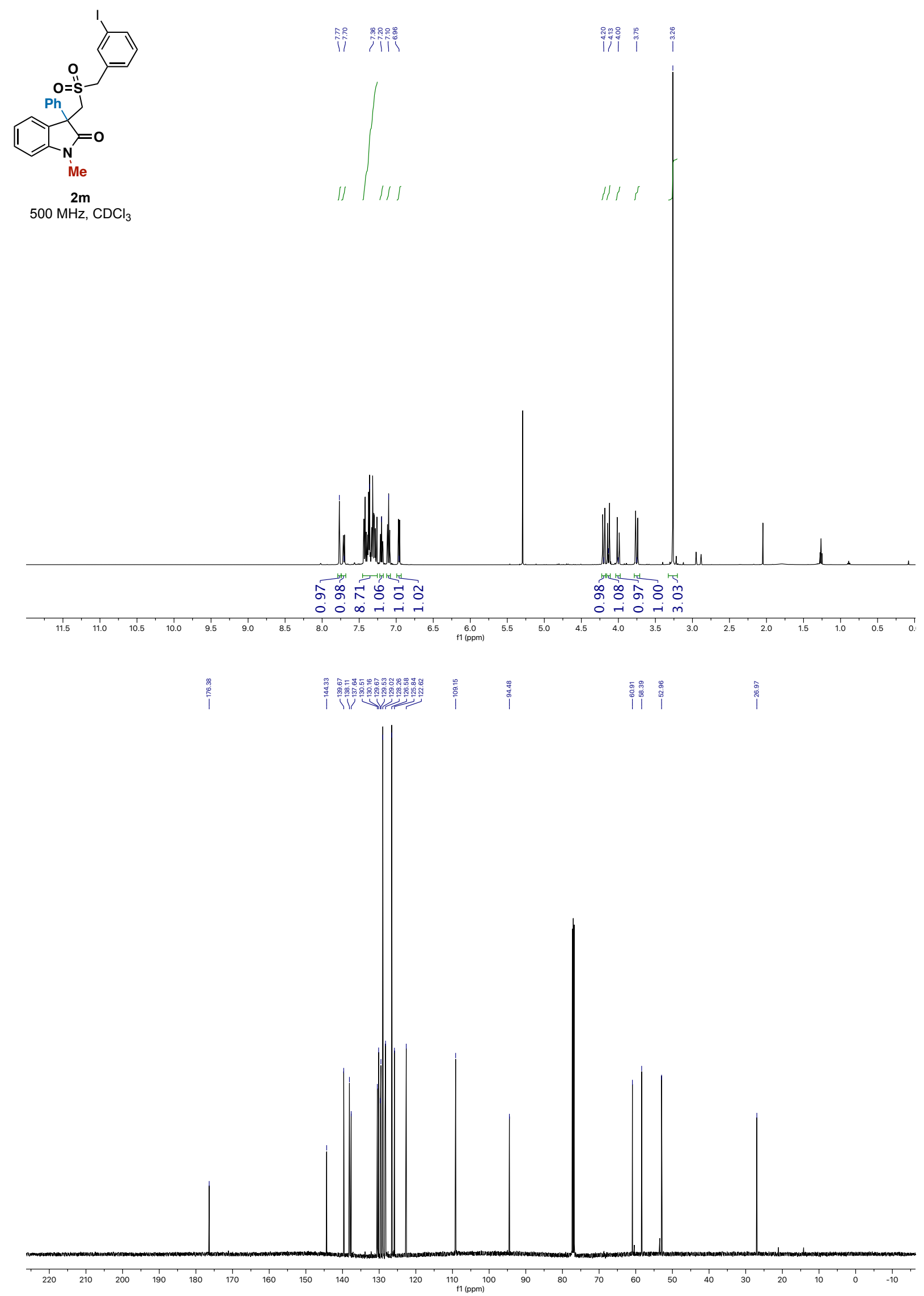

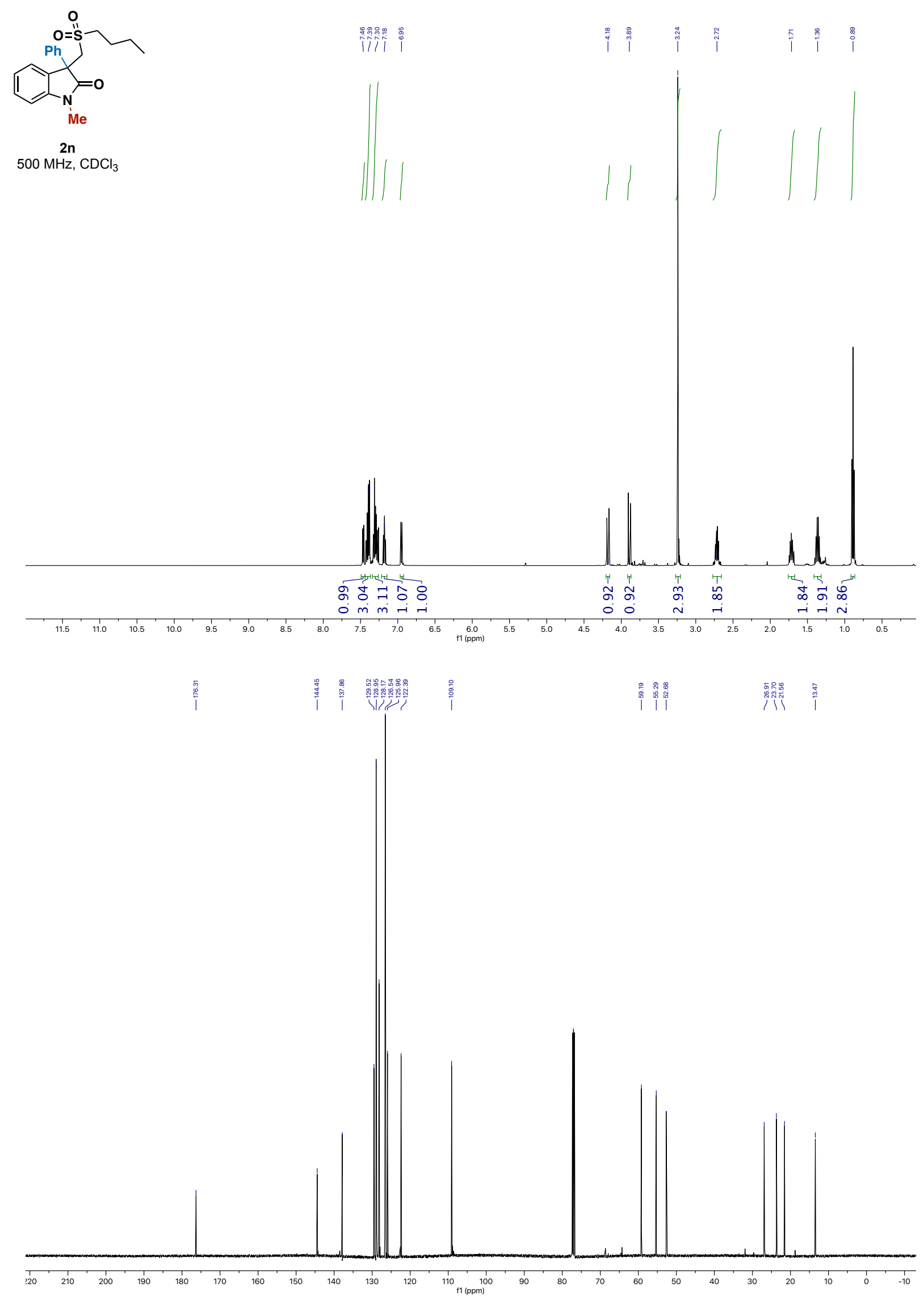


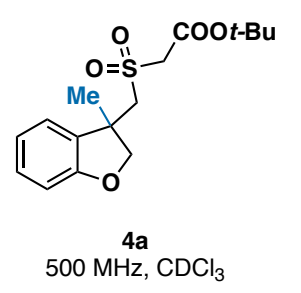

产

量 i

$\frac{8}{i \frac{3}{i}}$
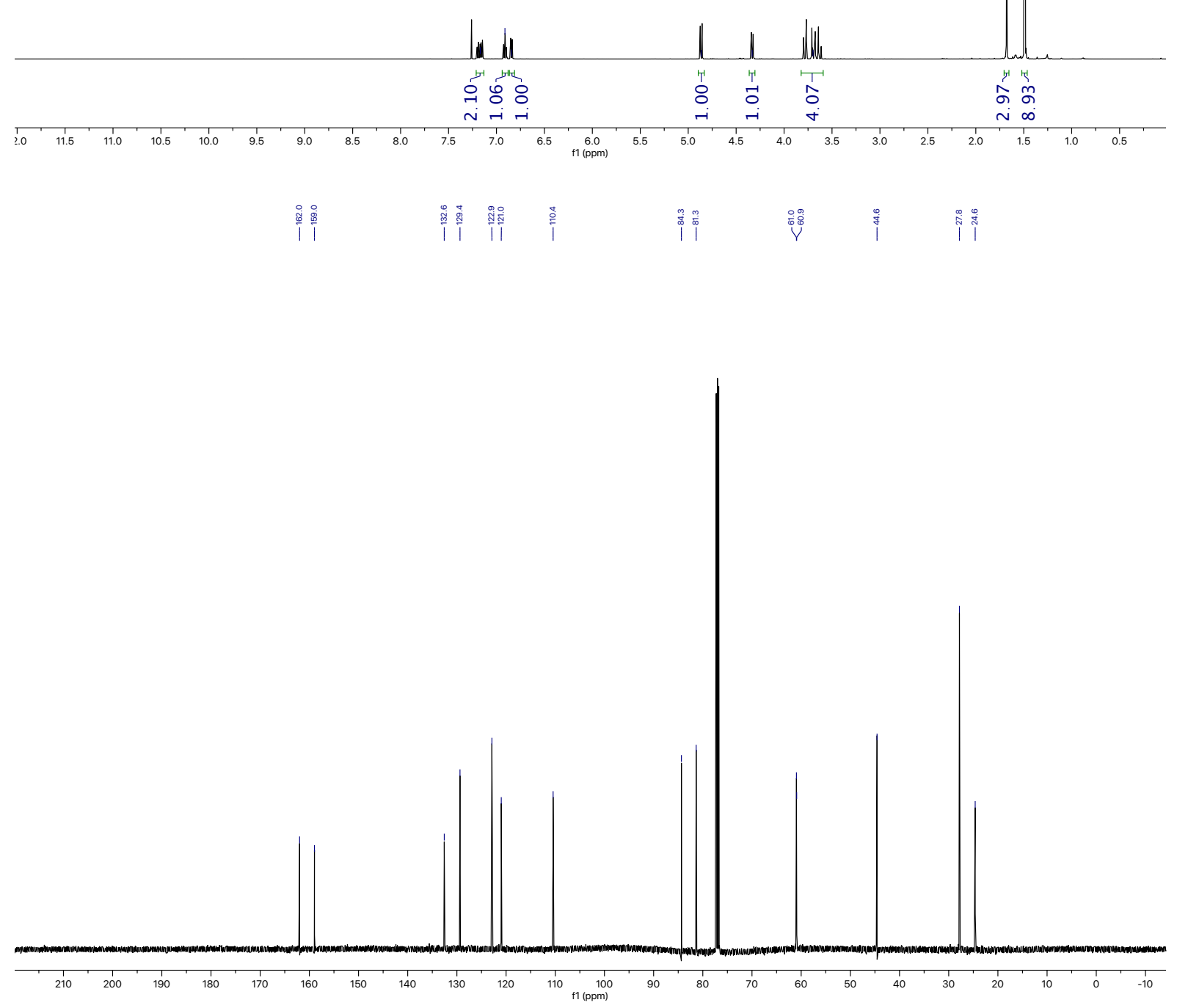

S32 

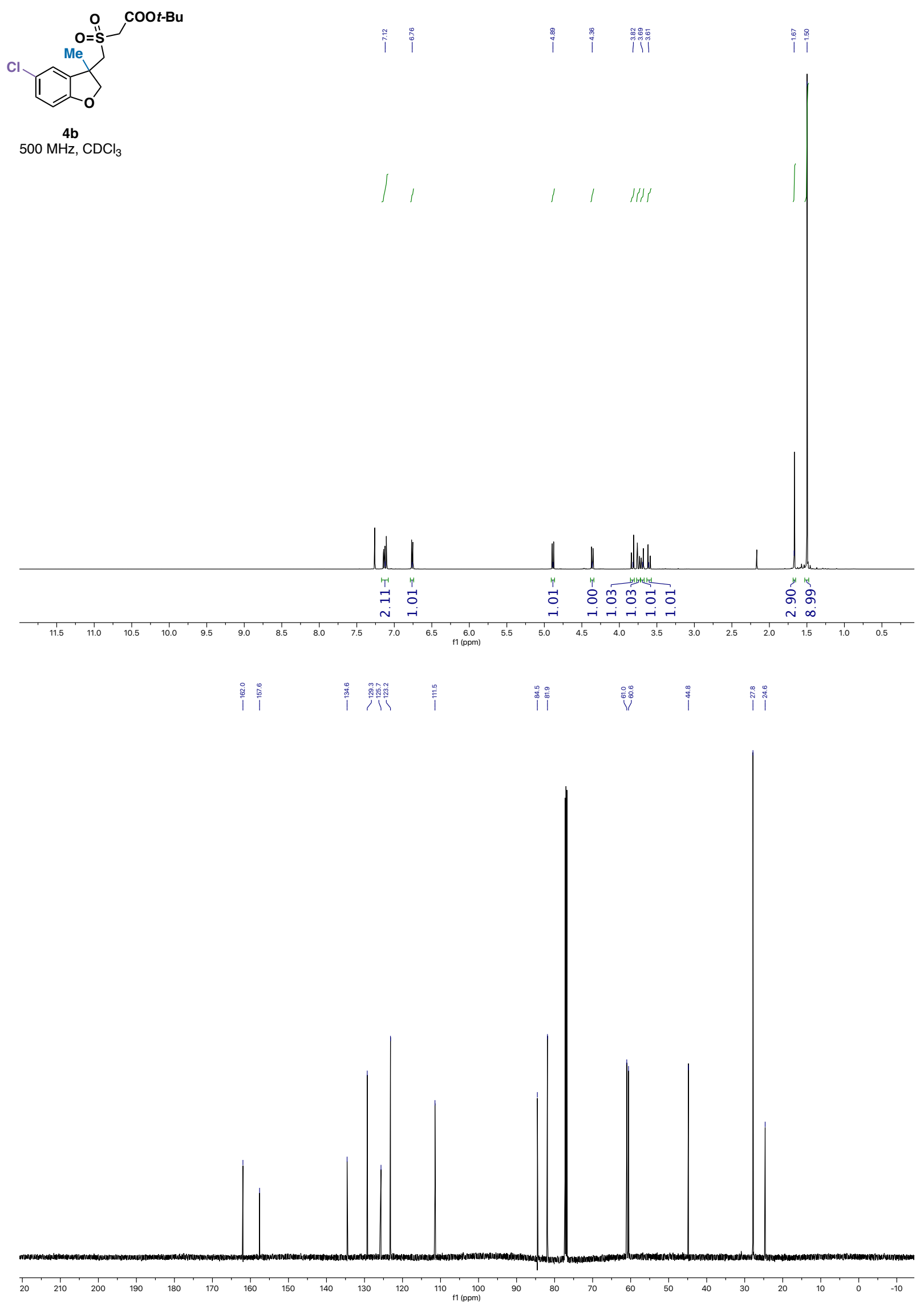

S33 

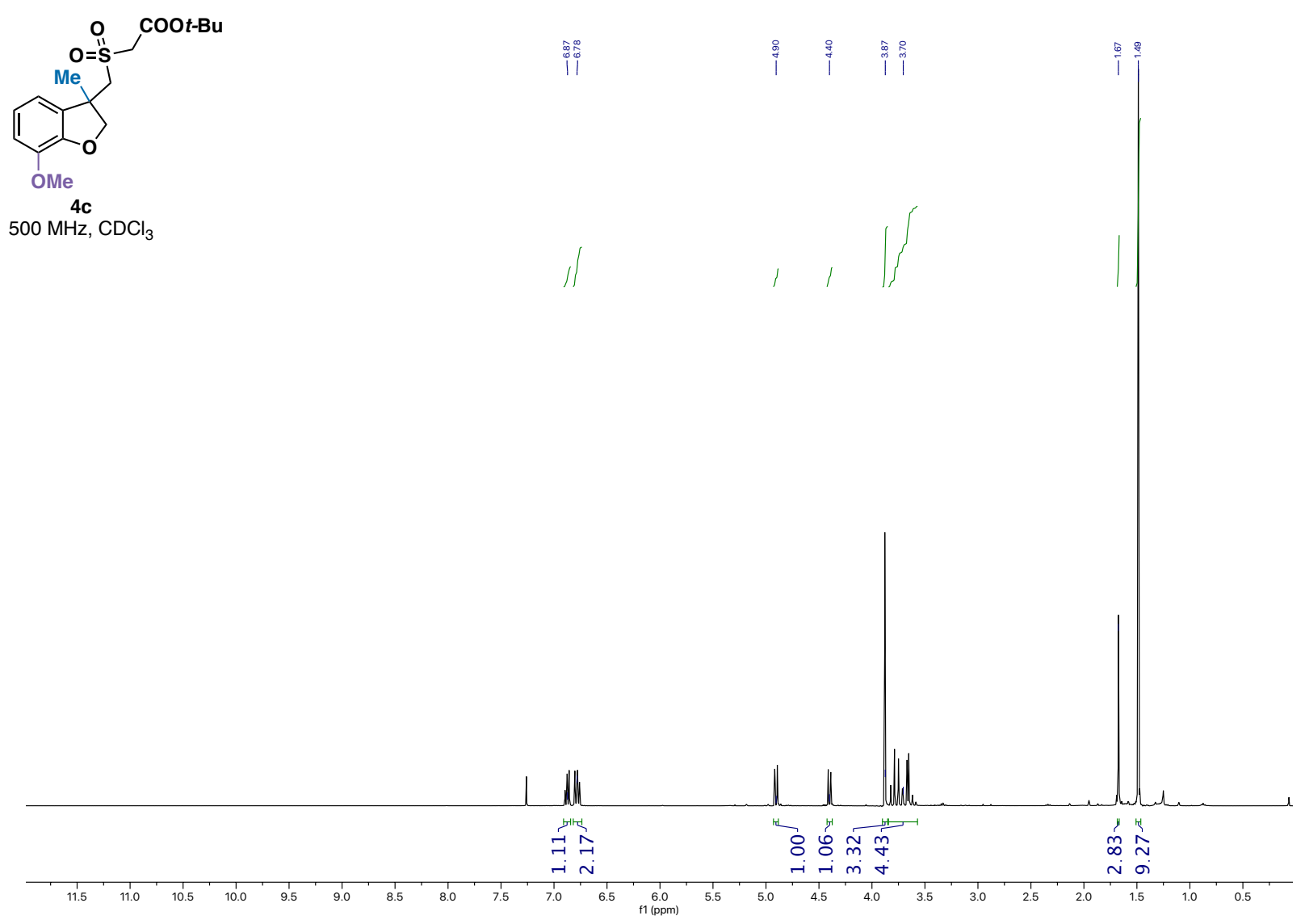

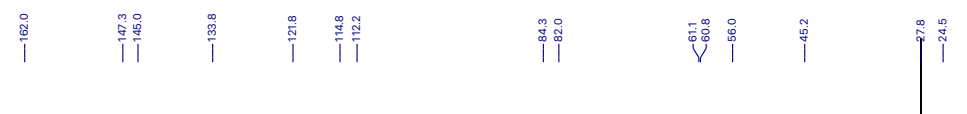
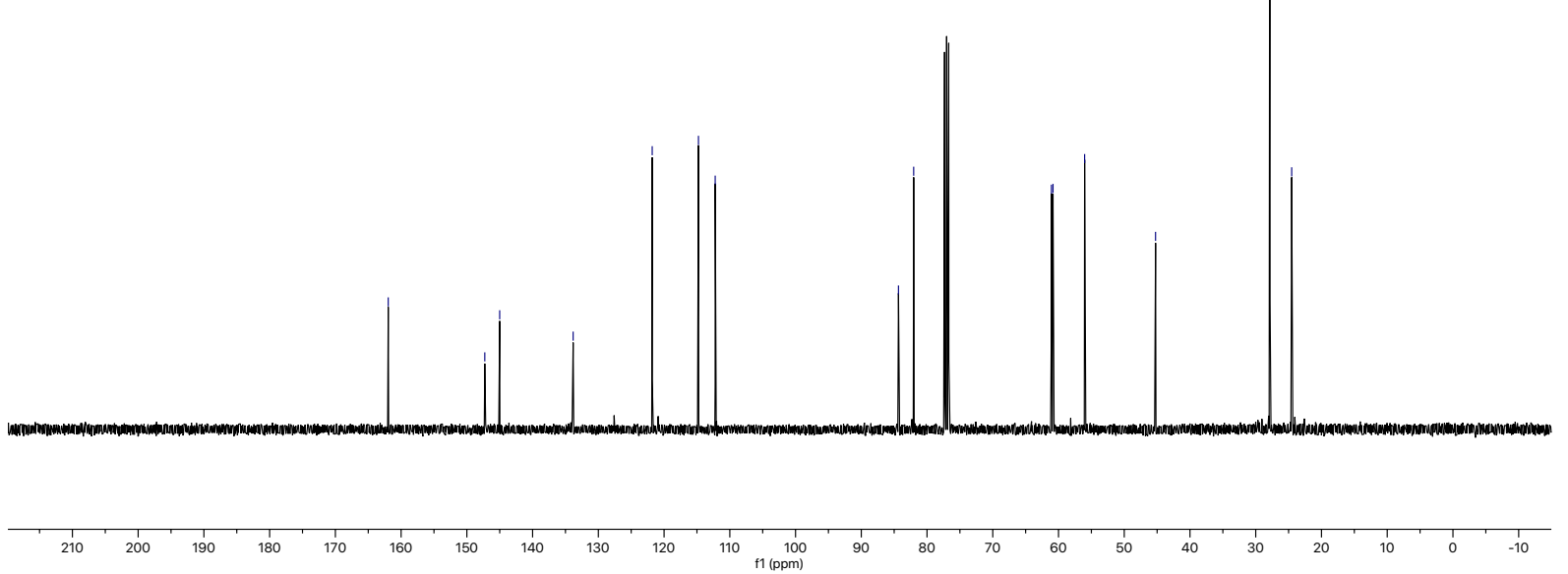


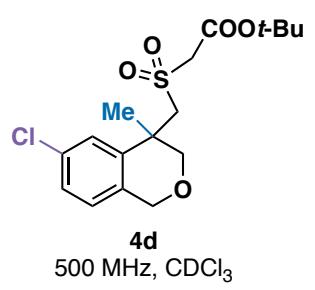

î

辛辛

111

1 11

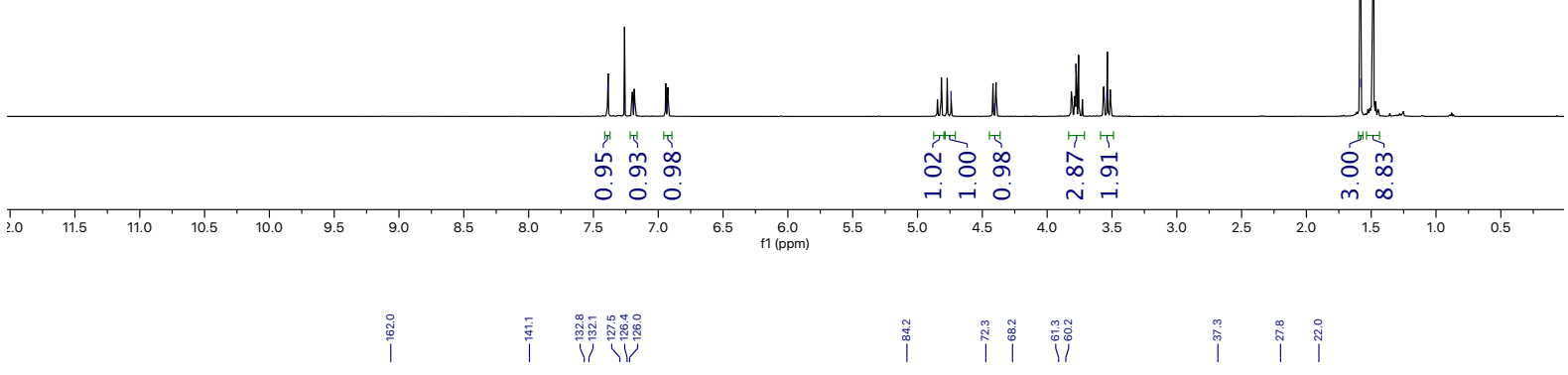

然

满

喁

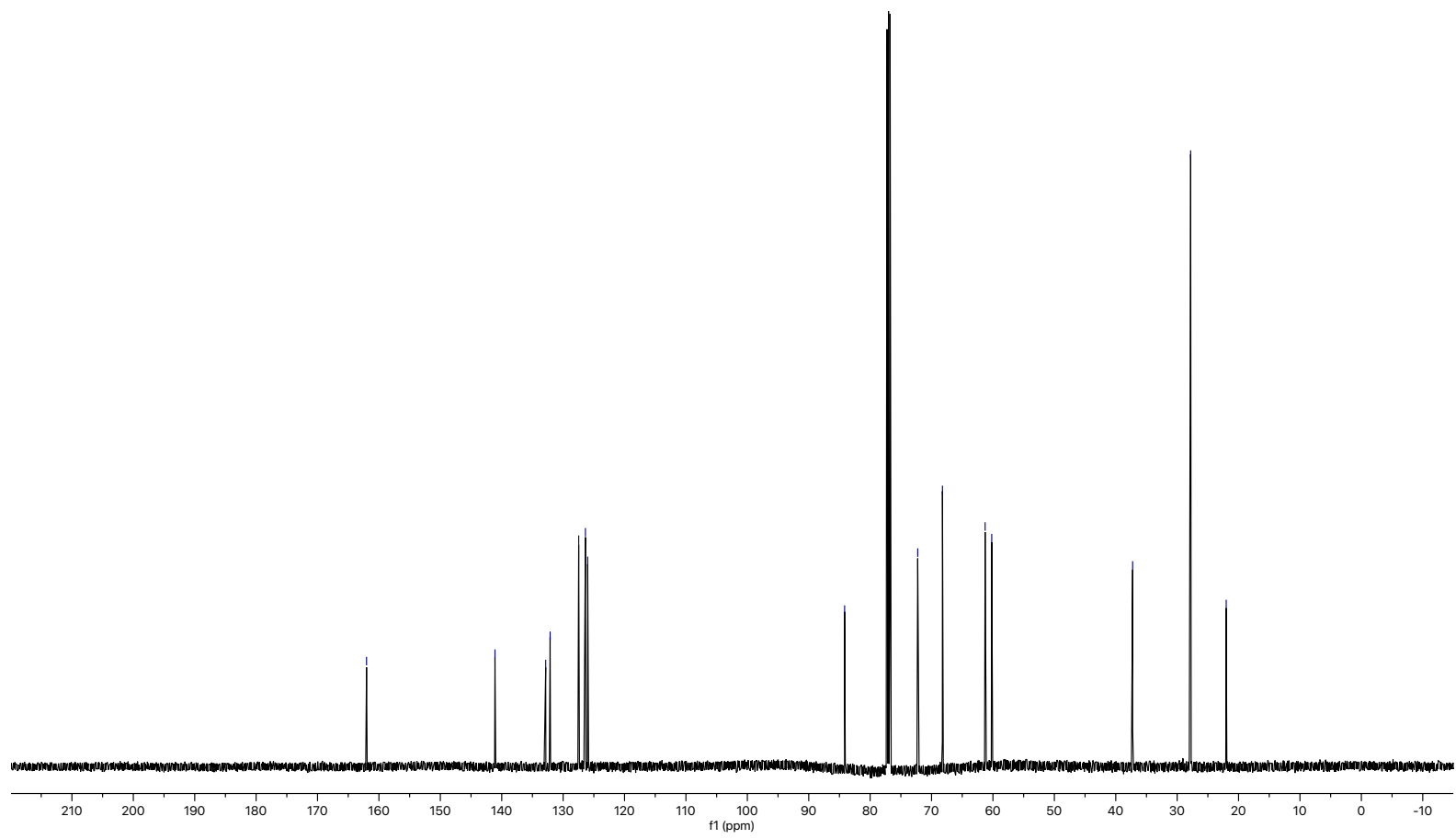

S35 


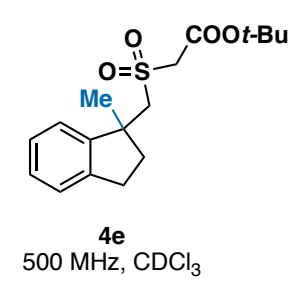

$500 \mathrm{MHz}, \mathrm{CDCl}_{3}$

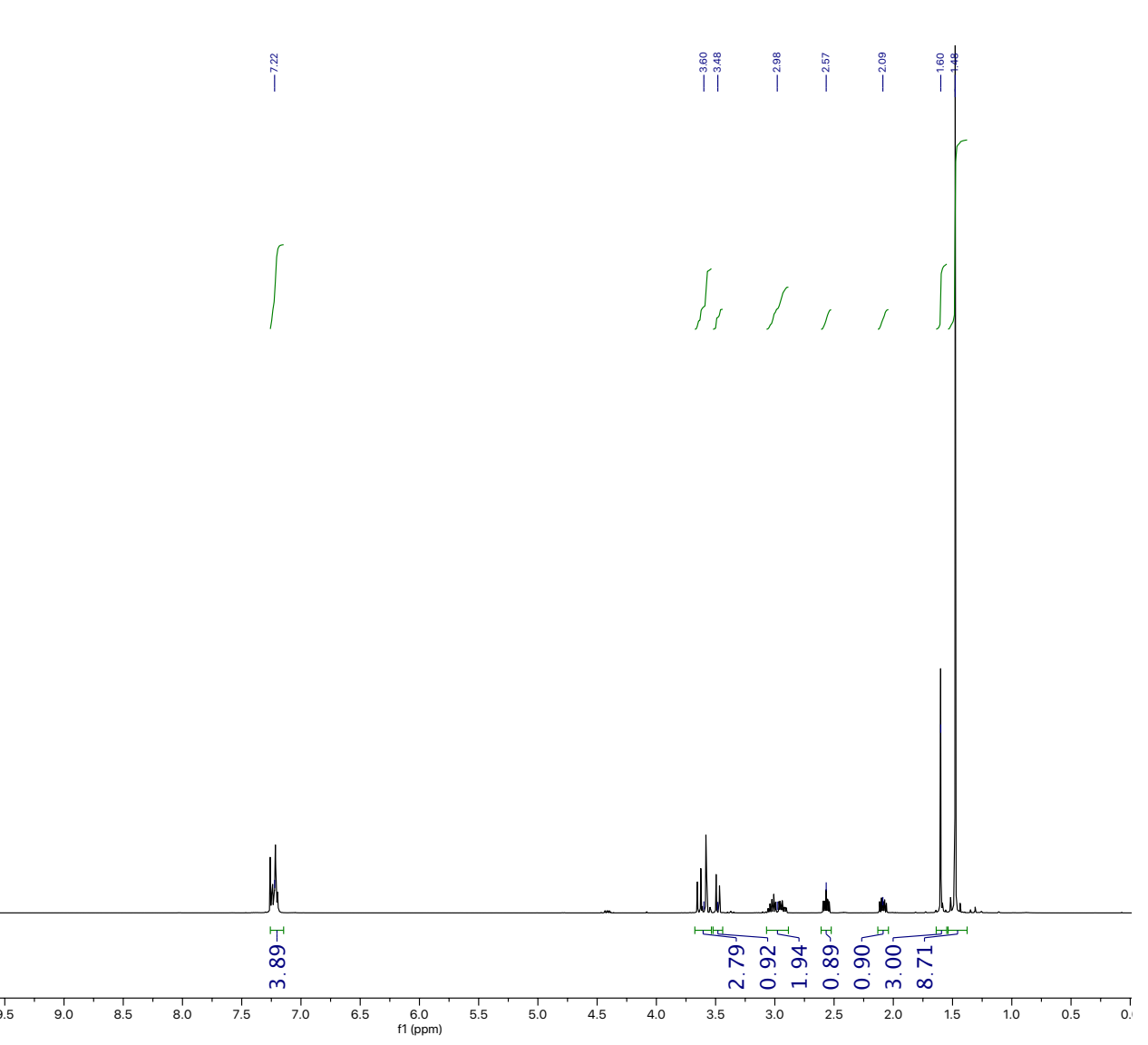

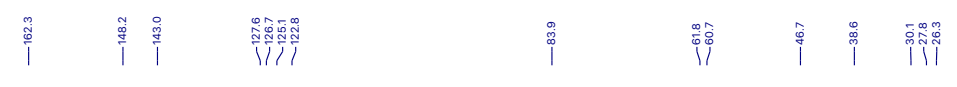

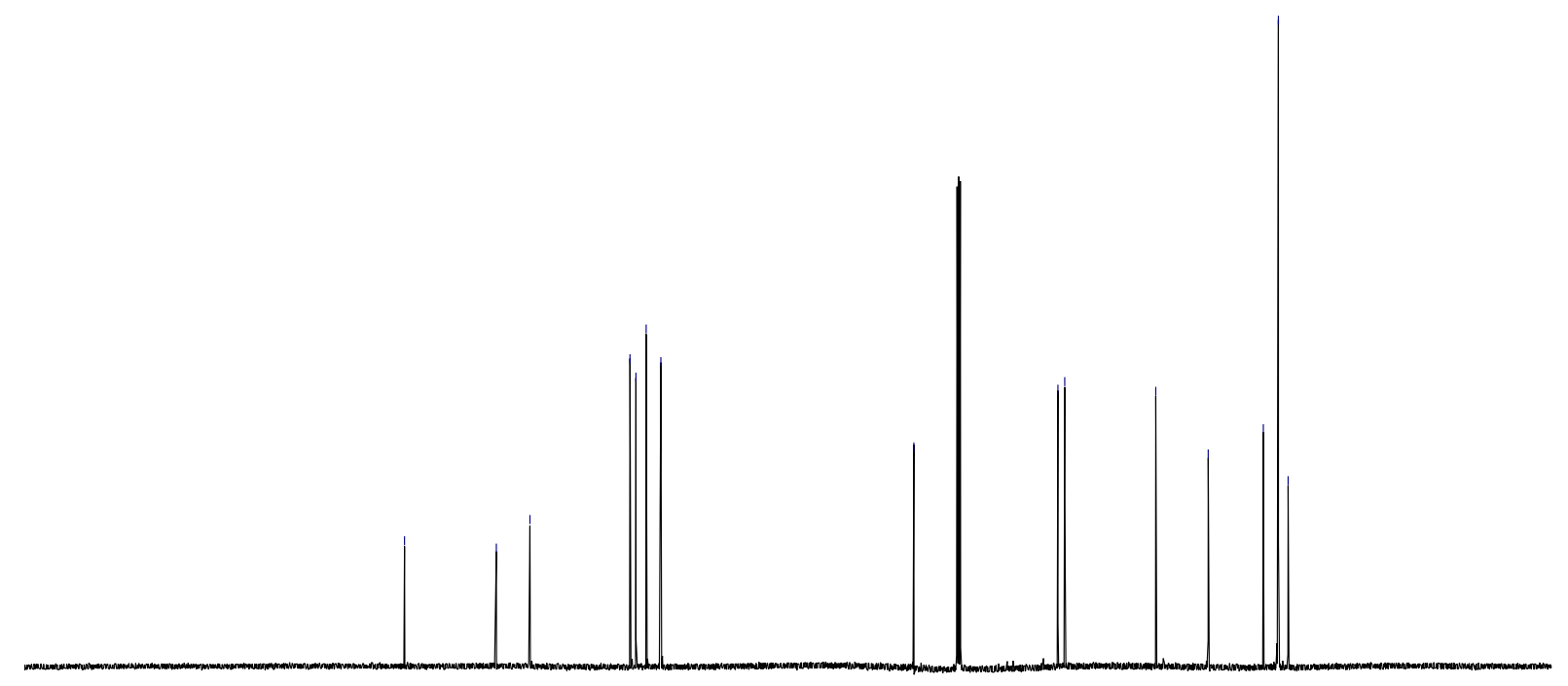

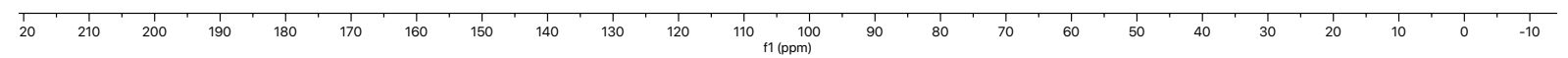



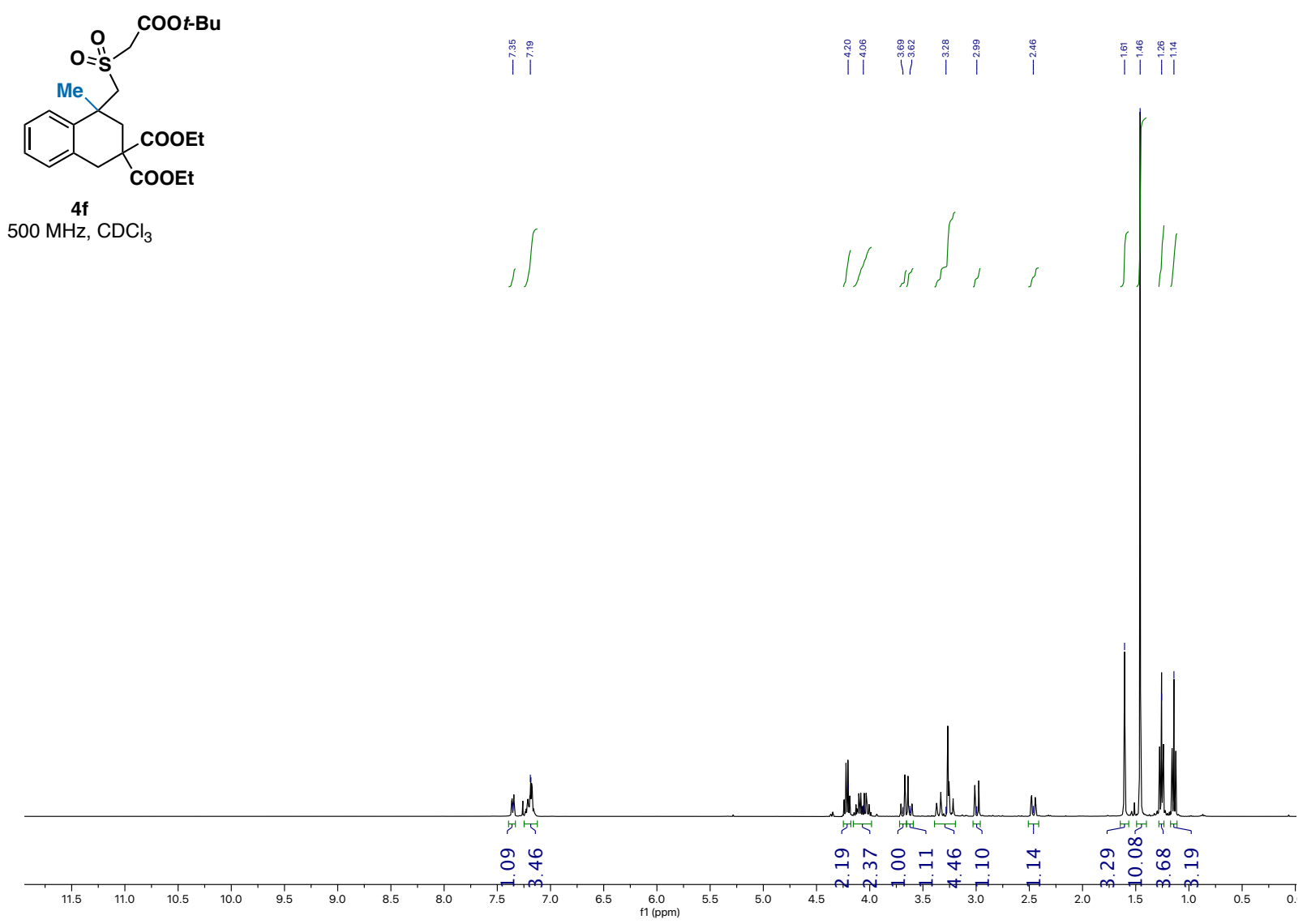

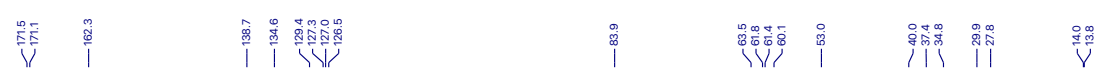

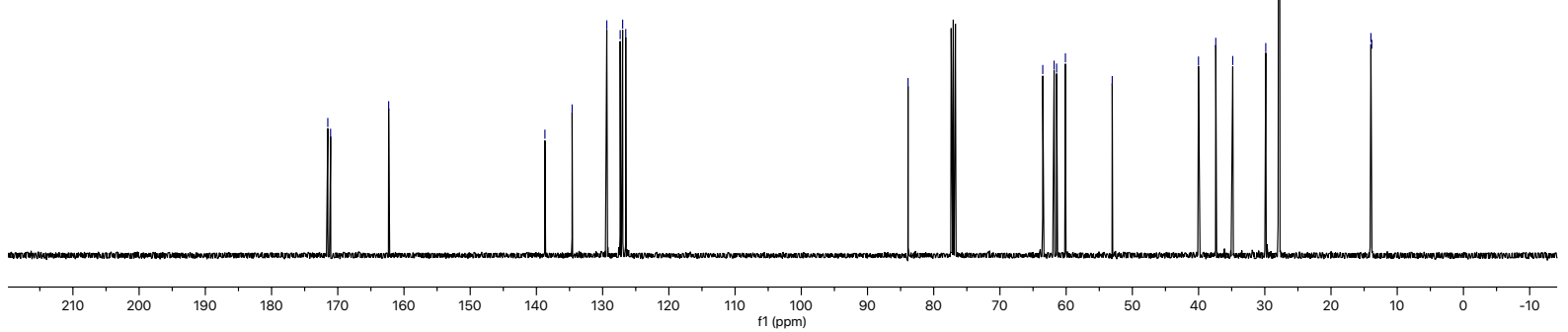



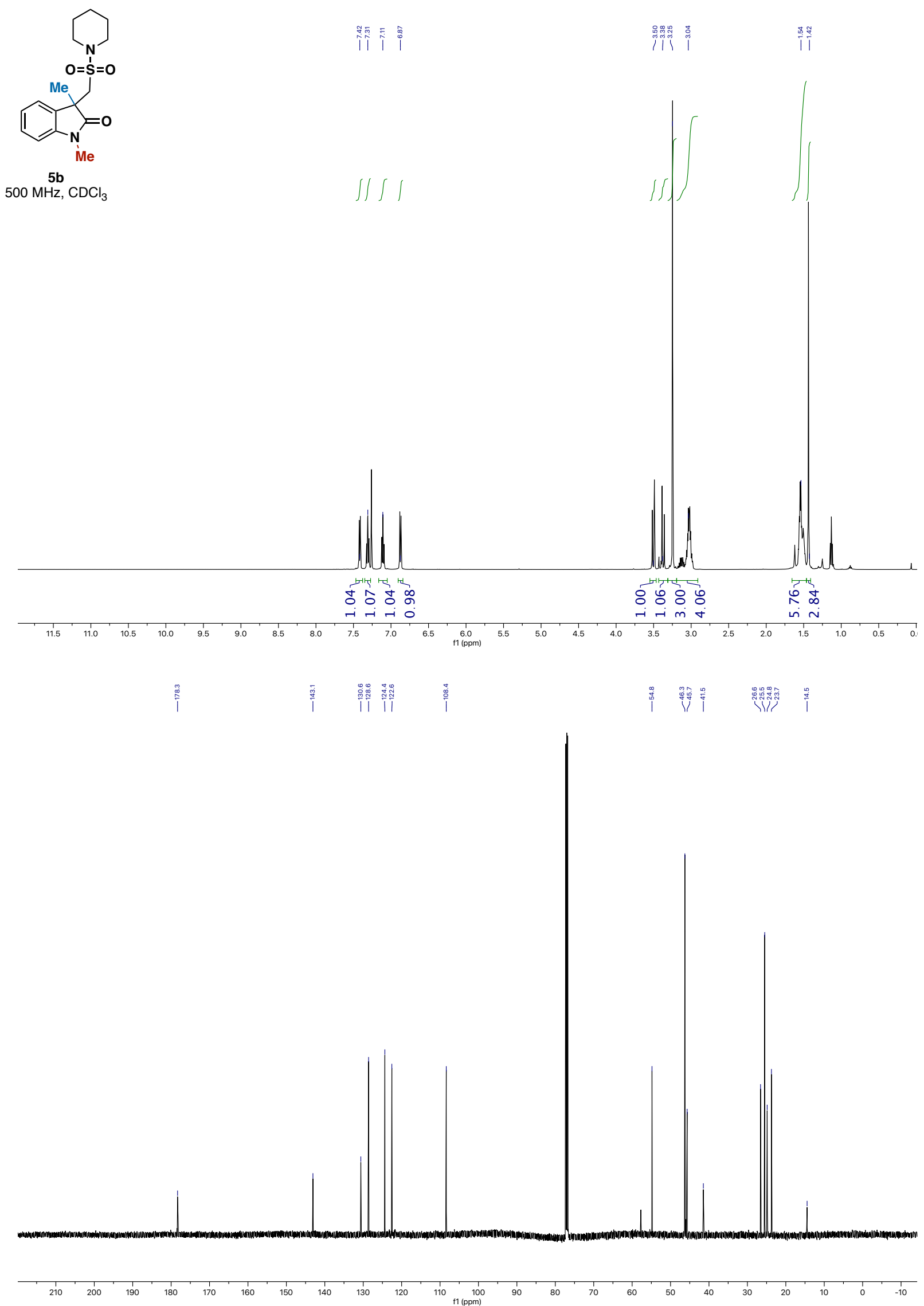


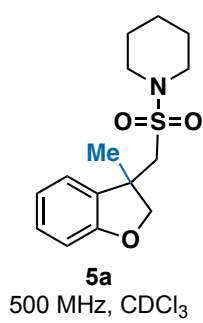

徐要祭
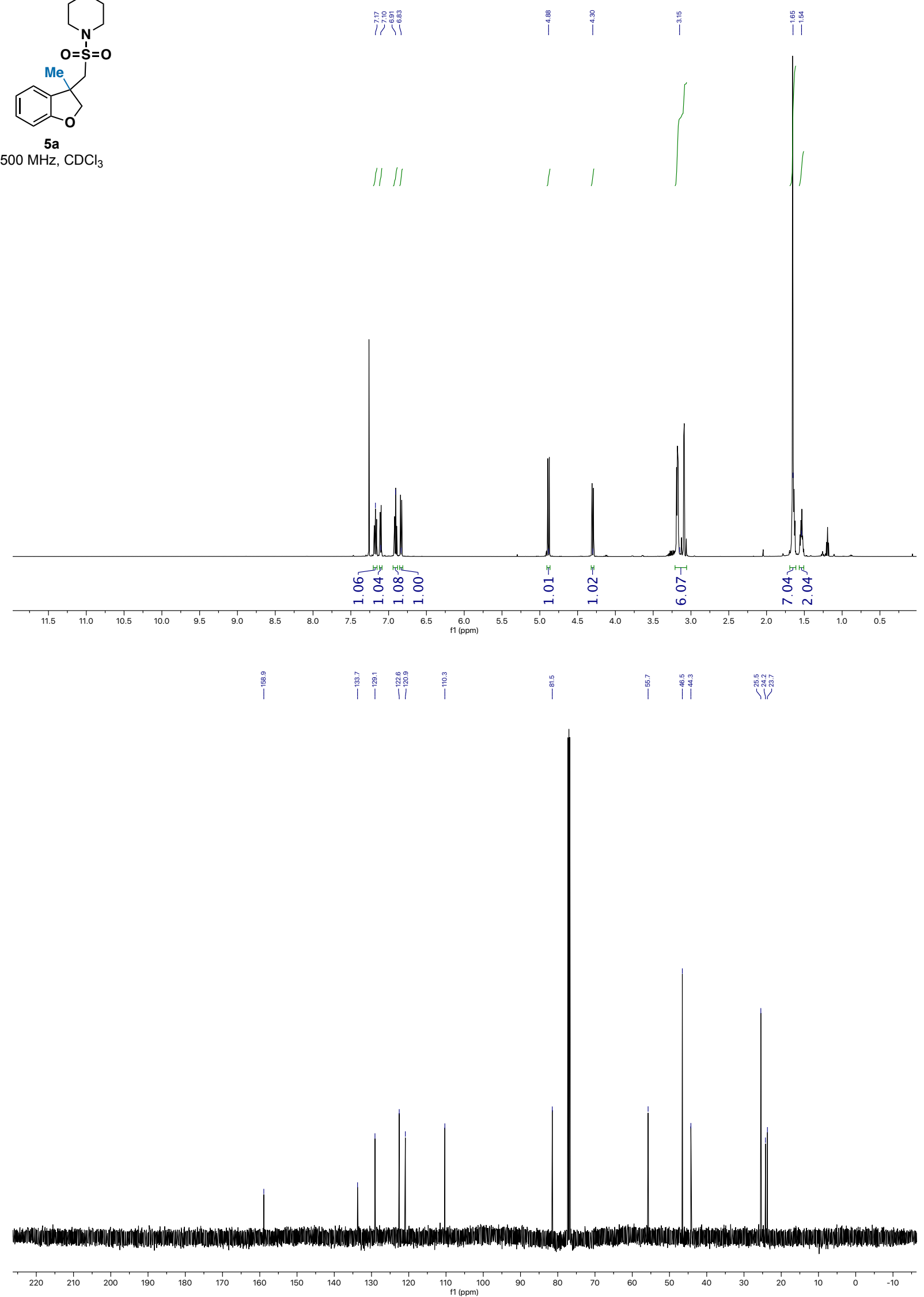

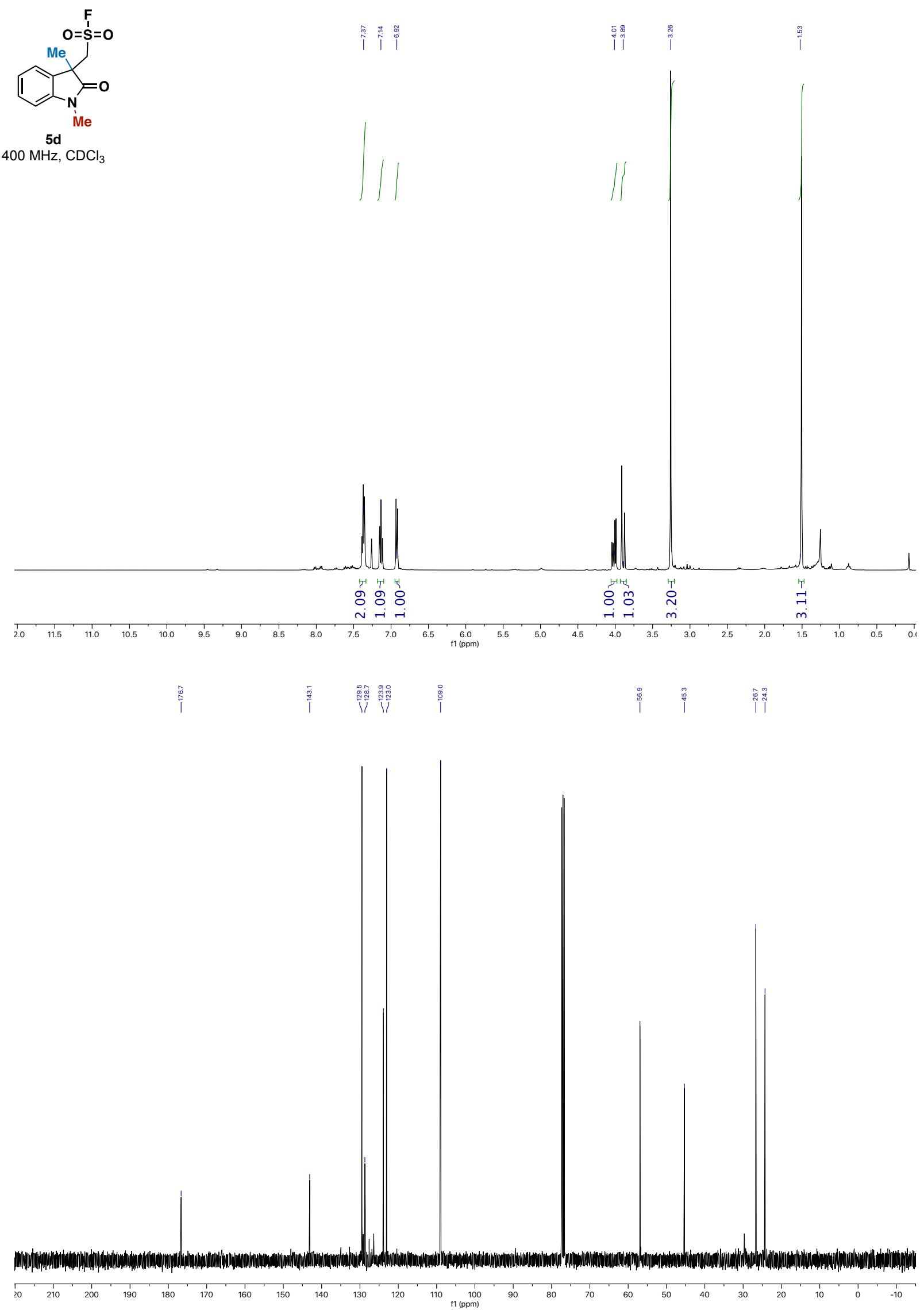
${ }^{19} \mathrm{~F} \mathrm{NMR} 400 \mathrm{MHz}, \mathrm{CDCl}_{3}$

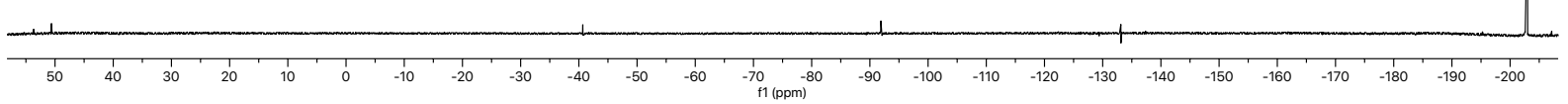



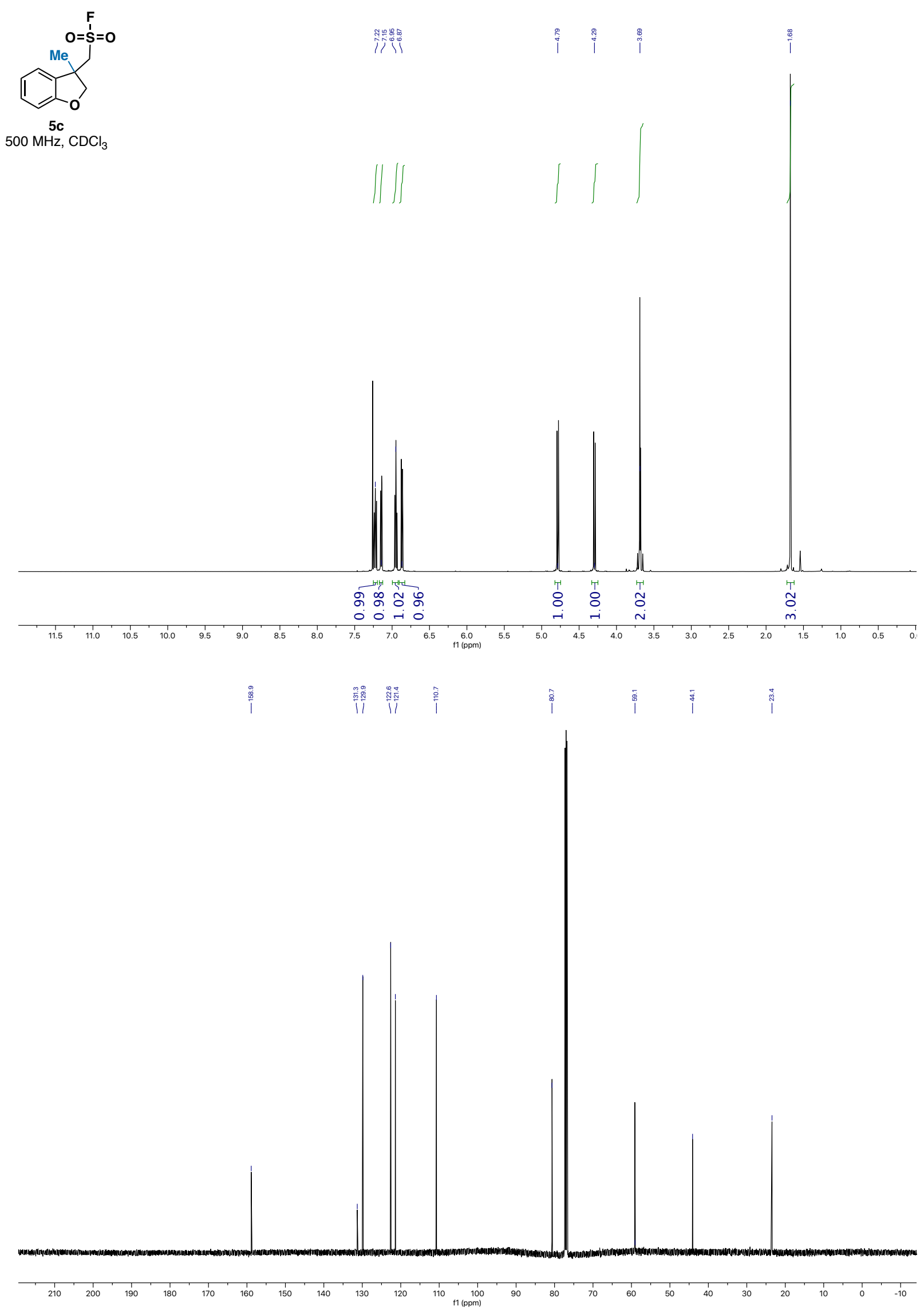
${ }^{19} \mathrm{~F} \mathrm{NMR} 400 \mathrm{MHz}, \mathrm{CDCl}_{3}$

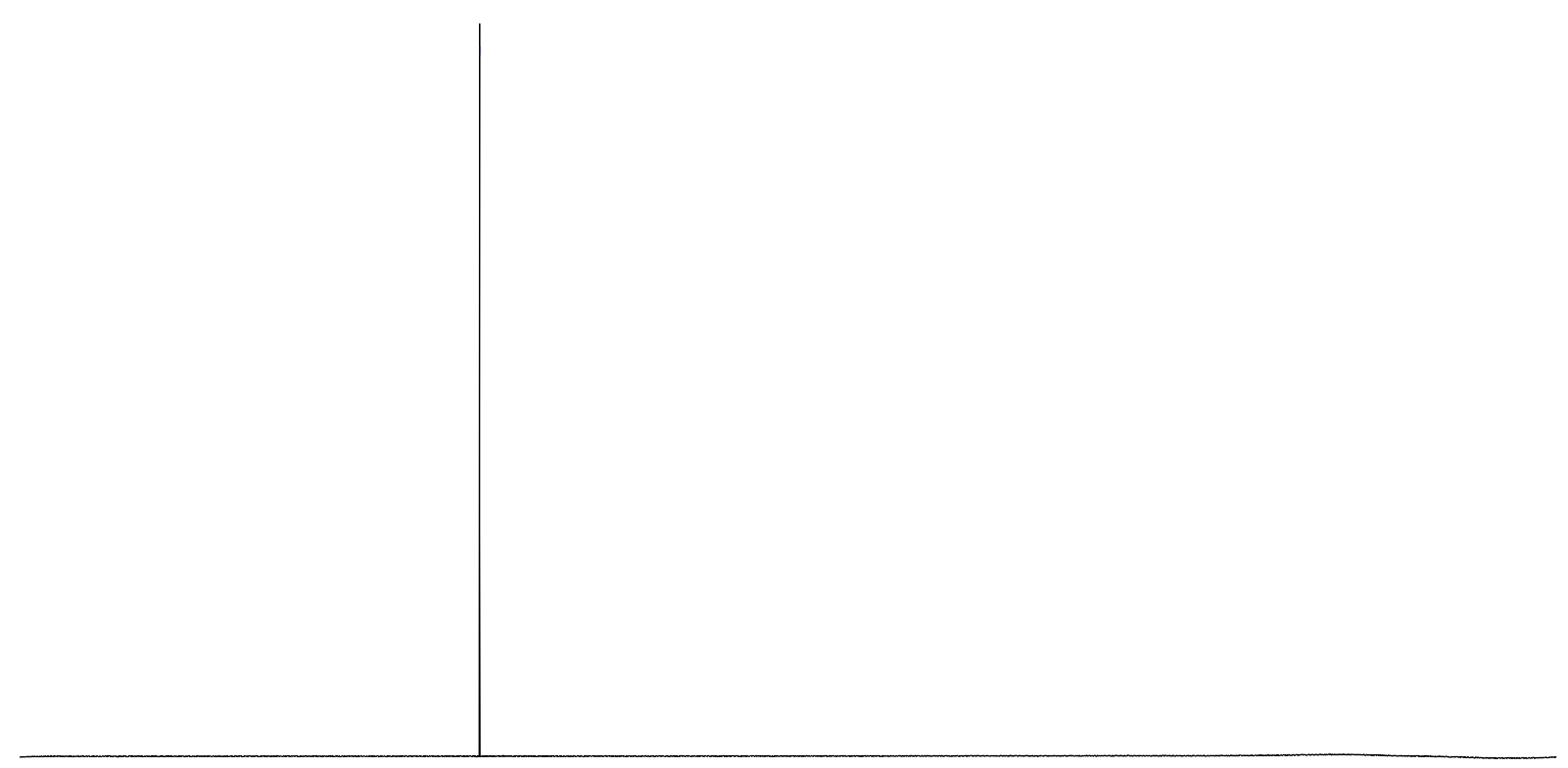

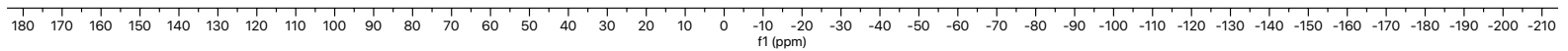




\section{References}

1. Van Mileghem, S.; De Borggraeve, W. M., A Convenient Multigram Synthesis of DABSO Using Sodium Sulfite as SO2 Source. Org. Process. Res. Dev. 2017, 21, $785-$ 787.

2. Feng, Y. X.; Yang, S. M.; Zhao, S.; Zhang, D. P.; Li, X. J.; Liu, H.; Dong, Y. H.; Sun, F. G., Nickel-Catalyzed Reductive Aryl Thiocarbonylation of Alkene via Thioester Group Transfer Strategy. Org. Lett. 2020, 22, 6734-6738.

3. Yoon, H.; Lossouarn, A.; Landau, F.; Lautens, M., Pd-Catalyzed Spirocyclization via CH Activation and Benzyne Insertion. Org. Lett. 2016, 18, 6324-6327.

4. He, J.; Xue, Y. H.; Han, B.; Zhang, C. Z.; Wang, Y.; Zhu, S. L., Nickel-Catalyzed Asymmetric Reductive 1,2-Carboamination of Unactivated Alkenes. Angew. Chem. Int. Ed. 2020, 59, 2328-2332.

5. Gao, Y.; Xiong, W. F.; Chen, H. J.; Wu, W. Q.; Peng, J. W.; Gao, Y. L.; Jiang, H. F., PdCatalyzed Highly Regio- and Stereoselective Formation of C-C Double Bonds: An Efficient Method for the Synthesis of Benzofuran-, Dihydrobenzofuran-, and IndolineContaining Alkenes. J. Org. Chem. 2015, 80, 7456-7467.

6. Wollenburg, M.; Bajohr, J.; Marchese, A. D.; Whyte, A.; Glorius, F.; Lautens, M., Palladium-Catalyzed Disilylation and Digermanylation of Alkene Tethered Aryl Halides: Direct Access to Versatile Silylated and Germanylated Heterocycles. Org. Lett. 2020, 22, 3679-3683.

7. Lu, Z. Y.; Hu, C. M.; Guo, J. J.; Li, J.; Cui, Y. X.; Jia, Y. X., Water-Controlled Regioselectivity of Pd-Catalyzed Domino Reaction Involving a C-H Activation Process: Rapid Synthesis of Diverse Carbo- and Heterocyclic Skeletons. Org. Lett. 2010, 12, 480483. 